\title{
Horizontal and Vertical Analysis of SAMSUNG Enterprise for the Years 2015-2016 and 2017-2018
}

\author{
Blerona Shala ${ }^{1}$, Abetare Prebreza ${ }^{1} \&$ Berim Ramosaj $^{2}$ \\ ${ }^{1} \mathrm{PhD}$ Candidate at University of Pristina "Hasan Pristina", Faculty of Economics, Kosovo \\ 2 Professor at University of Pristina "Hasan Pristina”, Faculty of Economics, Kosovo \\ Correspondence: Abetare Prebreza, University of Pristina "Hasan Pristina”, Faculty of Economics, Kosovo
}

Received: February 23, 2020; Accepted: March 8, 2021; Published: March 22, 2021

\begin{abstract}
The paper reflects our study about the horizontal and vertical analysis of financial statements. In this small volume we have tried to give meaningful, close and clear explanations. We have tried to methodologically elaborate the task in a gradual way. The paper is worked in several parts for the purpose of easier understanding.

The paper presents the original data of the financial statements, which elaborates the horizontal and vertical analysis of the balance sheet and the SAP for the years 2015-2015 and 2017-2018, in the next part is presented our commentary on the findings of the analysis for the above years. For this paper, SPSS software was used as a tool to interpret the results obtained from the questionnaire that was formulated for this paper.
\end{abstract}

Keywords: enterprise, financial statement, horizontal analysis, vertical analysis

\section{Theoretical Framework}

Users of financial statement information include managers, creditors, shareholders, investors, and regulatory agencies. These individuals and organizations can be divided into two groups both internal and external according to their interests in financial statement information (Wild, 2008).

Their purposes for using accounting information are different from each other. External users include shareholders, clients, regulators, lenders, governments, and other suppliers who are not directly involved in running an organization. Internal users include managers and employees who are directly involved in running and managing the organization (Brigham, 2015).

The purpose of the income statement is to show how profitable the firm has been during a given accounting period. This overview reports a summary of how the business generates its income and expenses through operating and non-operating activities (Weygandt, Keiso and Kimmel 2008, 2007). A result of this statement is given as profit or loss. The income statement is the most important ratio that investors, creditors and analysts are interested in (Kimmel 2001).

\section{Financial Statement Analysis Tools}

Various tools are used to evaluate the financial statements. Financial statement analysis consists of applying analytical tools and techniques to financial statements and other relevant data to obtain useful information. Three of the most common tools of financial statement analysis are: horizontal analysis, vertical analysis and ratio analysis (Wild, 2008).

\subsection{Horizontal Analysis}

Horizontal analysis, also called trend analysis, is a tool for estimating a series of financial statement data over a period of time. Its purpose is to investigate whether an increase and decrease has occurred. Analysis is mainly used in comparisons within the enterprise (Hermanson, Edwards and Salmonson, 1989).

The advantage of horizontal analysis is that changes can be expressed in quantities as well as in percentages. Horizontal analysis of period-to-period changes is relatively straightforward and quite useful (Keiso and Kimmel 2000). However, complications can occur during the calculation. If an item has no value in a base year or the previous year and a value in the following year, no percentage change can be calculated. If a negative amount appears at the base or previous year or period and a positive amount exists the following year, no percentage change can be calculated (Weygandt, 2001). 
Horizontal analysis analyzes the relationship between revenue and expense growth, meaning the increase in profits in a year, and possibly the increase in assets, liabilities and capital of companies (Joy Elly, 2017). The balance sheet and profit and loss account are diligently analyzed. Balance sheet analysis is particularly interested in the source that enhances the assets of the enterprise.

When investing in company ownership, the structure investor is particularly interested in the condition of its assets, capital and liabilities, and that there is a logical link between the increase in its assets, liabilities or equity (Joy Elly, 2017). The investor is particularly interested in whether the company's management uses horizontal financing policies. The most popular financing rule is the golden rule of banking 8 "Problems between assets and resources are expensive in small enterprises" (Atrill and McLaney, 2002).

\subsection{Vertical Analysis}

Vertical analysis is a tool that consists of studying a single financial statement in which each item is expressed as a percentage of a significant total. The use of vertical analysis is particularly useful in analyzing income statement data, such as the percentage of the cost of goods sold in sales (Hermanson, Edwards, \& Salmonson 1989,781).

Comparison of current assets with current liabilities or comparisons of debt to equity for a period of time are examples of vertical analysis (Kieso, 2013).

Thus, vertical analysis can be done in the following ways:

- By preparing joint size statements of two similar units

- Preparing the joint size statement of different years of the same business unit (Kieso, 2013).

Vertical analysis describes each amount of the financial statements as a percentage of another item. Vertical balance sheet valuation means that any amount belonging to the statement resists to be a percentage of the total valuation, while vertical analysis of liabilities in the balance sheet means that any amount belonging to the liability resists to be the percentage of total liabilities. Vertical analysis of an income statement shows that each amount of the income statement is represented as a percentage of sales (Patel, 2009).

In the cash flow statement, total cash inflow is set as a parameter for each post that forms cash flow, and total cash outflow is set as a parameter for each post that forms cash outflow, then cash and cash equivalents specified as a parameter of each post that the cash form and its equivalents in the cash flow statement (Brigham, 2004).

\section{Methodology}

\subsection{Population and Samples}

The population of this research consists of a number of microfinance institutions that operate in the Republic of Kosovo. According to the information provided by the Central Bank of the Republic of Kosovo, there are fourteen (14) microfinance institutions operating in our country. Which have 118 branches and sub-branches distributed throughout the country, with approximately 1021 employees (referring to the data of February 10, 2020, from the official website of the Association of Microfinance Institutions in RKS - "www.amik.org"). The sample of this research consists of four microfinance institutions, respectively KEP Trust, Finca, AFK and KRK. This choice of sample is not completely random, because in order to have a more detailed overview of the problem we are dealing with, the sample should have been taken by organizations with the largest number of employees and the widest range. organizations have similar characteristics (in terms of, services, employees, geographical location), which helps to conduct a more detailed analysis, more likely to notice any changes, but also more likely to understand more essentially the full picture of this issue in our country.

\subsection{Instrument and Procedure}

The instrument used in this research is the questionnaire as the easiest tool to reach the respondents. Anonymity was promised and kept and the willingness to attend was very high. The questionnaire contains 10 closed questions where the evaluation is done based on the Liqueur scale with 1-5 options to choose from.

Data collection was completed during July 2020. The questions were understandable and the distribution was done mainly through e-mail and media networks.Pyetjet hulumtuese dhe hipotezat.

Through this paper we will try to clarify the inevitable connection of Horizontal and Vertical Analysis in discovering the current state of enterprises and the appropriate actions to be taken before financial decisions are made.

\subsection{Research Questions}

Below are the initiating questions where this paper is concentrated. The research questions are as follows: 
1. How would the increase in foreign investment, as Samsung did during the 2015-2016 period, affect the enterprise?

2. How would the increase in accounts receivable and the decrease in accounts payable affect the enterprise?

\subsection{Hypotheses}

From the research questions the hypotheses are derived:

1. The increase of foreign investments as Samsung did during the period 2015-2018, will have a positive impact on the growth of the enterprise

2. Increasing accounts receivable and reducing accounts payable in the enterprise, would positively affect the enterprise

\section{Original Data}

\subsection{Balance Sheet}

Figure 1. Balance sheet of Samsung until 2018

\begin{tabular}{|c|c|c|c|c|c|}
\hline Balance Sheet Items & Counting & Year 2015 & Year 2016 & Year 2017 & Year 2018 \\
\hline \multicolumn{6}{|l|}{ Assets } \\
\hline \multicolumn{6}{|l|}{ Money and its equivalents Current assets } \\
\hline Money and its equivalents & 1 & $13,716,517$ & $17,544,076$ & $15,431,422$ & $15,997,688$ \\
\hline Short-term financial instruments & 2 & $10,764,546$ & $16,243,056$ & $34,798,353$ & $39,602,713$ \\
\hline Available-for-sale financial assets & 3 & 612,426 & $1,175,309$ & $1,410,525$ & $3,122,255$ \\
\hline $\mathrm{A} / \mathrm{R}$ & 4 & $22,549,741$ & $24,903,927$ & $26,415,175$ & $26,821,018$ \\
\hline Advances & 5 & $1,340,947$ & $1,563,279$ & $1,827,147$ & $1,889,874$ \\
\hline Prepaid expenses & 6 & $2,174,832$ & $2,112,066$ & $2,343,362$ & $3,179,057$ \\
\hline Inventory & 7 & $14,673,434$ & $16,569,333$ & $18,132,160$ & $16,450,560$ \\
\hline Assets held for sale & 8 & 0 & 0 & $2,574,370$ & 613,177 \\
\hline Other current assets & 9 & 923,288 & $1,365,023$ & $2,023,680$ & $1,705,275$ \\
\hline Total current assets & 10 & $66,755,731$ & $81,476,069$ & $104,956,194$ & $109,381,617$ \\
\hline \multicolumn{6}{|l|}{ Non-current assets } \\
\hline Available-for-sale financial assets & 11 & $3,009,614$ & $4,882,060$ & $5,911,475$ & $12,033,351$ \\
\hline Investments in other enterprises (j.v) & 12 & $8,593,193$ & $8,202,305$ & $6,085,750$ & $4,970,515$ \\
\hline Land, factories and equipment & 13 & $57,925,451$ & $63,938,701$ & $71,540,214$ & $76,824,309$ \\
\hline Intangible assets & 14 & $3,132,514$ & $3,482,126$ & $3,772,008$ & $4,545,904$ \\
\hline deposits & 15 & 739,299 & 760,613 & 0 & 0 \\
\hline Prepayment of long-term expenses & 16 & $3,224,914$ & $3,282,120$ & $3,284,168$ & $4,613,970$ \\
\hline Income tax prepayment (profit) & 17 & $1,664,724$ & $2,349,062$ & $4,379,589$ & $4,299,986$ \\
\hline Other fixed assets & 18 & 412,746 & 678,919 & $2,927,627$ & $2,217,933$ \\
\hline Total non-current assets & 19 & $78,702,455$ & $87,575,906$ & $87,309,447$ & $98,374,079$ \\
\hline TOTAL PROPERTY & 20 & $145,458,186$ & $169,051,975$ & $192,265,641$ & $207,755,696$ \\
\hline \multicolumn{6}{|l|}{ Liabilities and Capital } \\
\hline \multicolumn{6}{|l|}{ Short-term liabilities } \\
\hline ACCOUNTS PAYABLE & 21 & $17,280,823$ & $15,768,229$ & $16,709,661$ & $7,518,480$ \\
\hline Short-term loans & 22 & $9,012,904$ & $7,883,253$ & $6,101,125$ & $7,627,338$ \\
\hline Unearned income & 23 & $1,354,433$ & $1,416,928$ & $1,616,899$ & $1,355,780$ \\
\hline Withholding tax & 24 & $1,601,223$ & 902,226 & $1,114,419$ & $1,103,482$ \\
\hline Accrual costs & 25 & $7,304,386$ & $8,864,864$ & $10,750,052$ & $12,232,143$ \\
\hline Profit tax payable & 26 & $1,178,973$ & $3,008,994$ & $3,208,583$ & $2,052,920$ \\
\hline Loans and bonds & 27 & 0 & 0 & $2,298,712$ & $1,689,624$ \\
\hline Current installment of long-term loans & 28 & 28,281 & 932,695 & 0 & 0 \\
\hline Provisions & 29 & $3,281,240$ & $4,719,310$ & $6,383,470$ & $5,691,565$ \\
\hline Liabilities held for sale & 30 & 0 & 0 & 0 & 26,898 \\
\hline Other short-term liabilities & 31 & 334,839 & 321,120 & 443,450 & $10,111,780$ \\
\hline Total current liabilities & 32 & $41,377,102$ & $43,817,619$ & $48,626,371$ & $49,410,010$ \\
\hline
\end{tabular}




\begin{tabular}{|c|c|c|c|c|c|}
\hline \multicolumn{6}{|l|}{ Long-term liabilities } \\
\hline & 33 & 0 & 0 & $1,242,365$ & $1,288,004$ \\
\hline Long-term loans & 34 & $1,195,149$ & $1,707,940$ & 933,495 & 96,581 \\
\hline LIABILITIES PAYABLE & 35 & 956,777 & $1,088,489$ & 998,537 & $2,433,999$ \\
\hline Net defined benefit obligations & 36 & 0 & 0 & $1,757,701$ & 191,262 \\
\hline Liabilities Other unsecured long-term bonds & 37 & $3,438,028$ & $3,382,530$ & 0 & 0 \\
\hline Pension liability & 38 & $1,044,896$ & $1,615,105$ & 0 & 0 \\
\hline Profit tax (income) payable & 39 & $2,178,547$ & $3,201,818$ & $5,697,310$ & $3,892,667$ \\
\hline Provisions & 40 & 339,112 & 381,411 & 436,771 & 474,295 \\
\hline Other long-term liabilities & 41 & 340,180 & 440,757 & $1,009,628$ & $1,427,368$ \\
\hline TOTAL OBLIGATIONS & 43 & $50,869,791$ & $55,635,669$ & $60,702,178$ & $59,214,186$ \\
\hline \multicolumn{6}{|l|}{ Equity } \\
\hline Ordinary shares & 45 & 726,400 & 726,400 & 737,276 & 739,097 \\
\hline Premium from shares & 46 & $4,111,561$ & $4,111,561$ & $4,173,119$ & $4,183,426$ \\
\hline Retained earnings & 47 & $91,142,630$ & $112,020,996$ & $140,813,307$ & $161,042,656$ \\
\hline Other reserves & 48 & $-5,446,639$ & $-7,649,185$ & $-8,963,398$ & $-12,092,132$ \\
\hline Other comprehensive income & 49 & 0 & 0 & & 76,091 \\
\hline Uncontrolled interest & 50 & $3,942,906$ & $4,094,997$ & $5,281,336$ & $5,610,775$ \\
\hline EQUITY & 51 & $\mathbf{9 4 , 5 8 8 , 3 9 5}$ & $113,416,306$ & $142,154,847$ & $159,673,399$ \\
\hline Preferential shares & 44 & 111,537 & 111,537 & 113,207 & 113,486 \\
\hline Total long-term liabilities & 42 & $8,297,540$ & $10,110,110$ & $\mathbf{1 2 , 0 7 5 , 8 0 7}$ & $9,804,176$ \\
\hline Total liabilities and capital & 52 & $145,458,186$ & $169,051,975$ & $202,857,025$ & $218,887,585$ \\
\hline
\end{tabular}

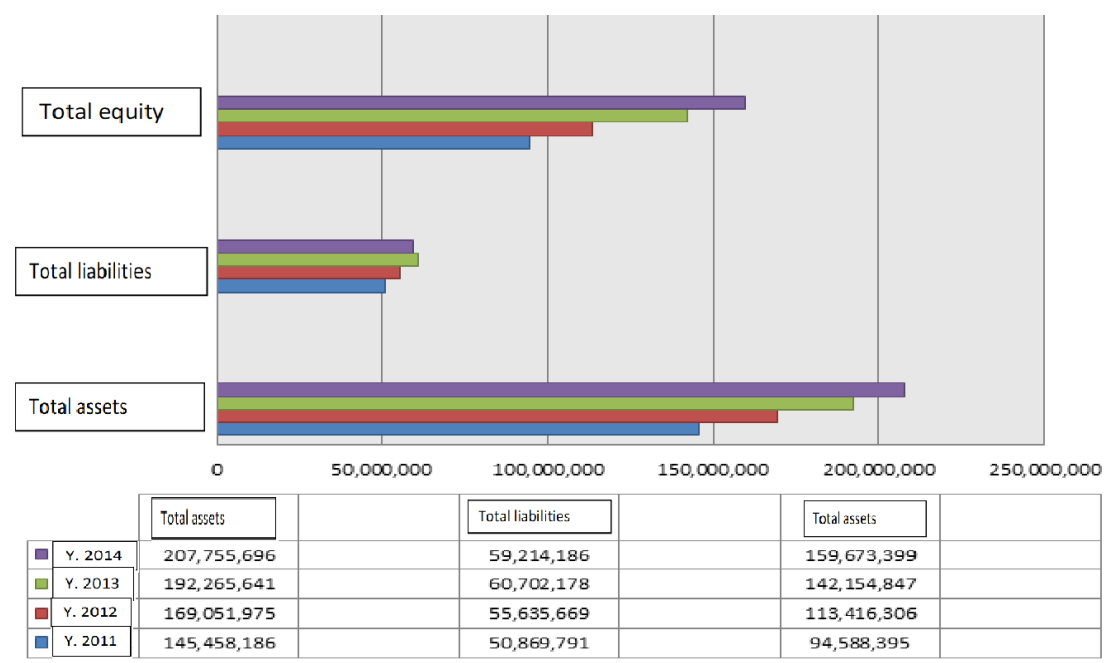

Chart 1. Samsung Balance Sheet

\subsection{Income and Expense Statement}

Table 1. Statement of income and expenses until 2018

\begin{tabular}{llrrrr}
\hline ITEMS & Counting & \multicolumn{1}{c}{ Year $\mathbf{2 0 1 5}$} & \multicolumn{1}{c}{ Year 2016 } & Year 2017 & \multicolumn{1}{c}{ Year 2018 } \\
\hline Revenues from sales & $\mathbf{5 3}$ & $\mathbf{1 5 4 , 0 4 8 , 8 9 5}$ & $\mathbf{1 8 7 , 7 5 4 , 2 8 3}$ & $\mathbf{2 1 6 , 7 0 8 , 6 7 7}$ & $\mathbf{1 9 5 , 8 8 2 , 9 5 5}$ \\
Cost of goods sold & $\mathbf{5 4}$ & $(104,700,887)$ & $(118,244,730)$ & $(130,480,72)$ & $(121,856,939)$ \\
Gross Profit & $\mathbf{5 5}$ & $\mathbf{4 9 , 3 4 8 , 0 0 8}$ & $\mathbf{6 9 , 5 0 9 , 5 5 3}$ & $\mathbf{8 6 , 2 2 7 , 9 5 2}$ & $\mathbf{7 4 , 0 2 6 , 0 1 6}$ \\
Operating expenses & $\mathbf{5 6}$ & $(34,742,191)$ & $(42,388,520)$ & $(51,370,553)$ & $(50,253,744)$ \\
Operating Profit & $\mathbf{5 7}$ & $\mathbf{1 4 , 6 0 5 , 8 1 7}$ & $\mathbf{2 7 , 1 2 1 , 0 3 3}$ & $\mathbf{3 4 , 8 5 7 , 3 9 9}$ & $\mathbf{2 3 , 7 7 2 , 2 7 2}$ \\
Financial income & $\mathbf{5 8}$ & $1,444,895$ & 808,215 & $1,469,509$ & $2,707,288$ \\
Profit before tax & $\mathbf{5 9}$ & $\mathbf{1 6 , 0 5 0 , 7 1 2}$ & $\mathbf{2 7 , 9 2 9 , 2 4 8}$ & $\mathbf{3 6 , 3 5 3 , 9 0 8}$ & $\mathbf{2 6 , 4 7 9 , 5 6 0}$ \\
Income tax expense & $\mathbf{6 0}$ & $(3,204,999)$ & $(5,666,822)$ & $(7,476,087)$ & $(4,256,366)$ \\
Profit of the year & $\mathbf{6 1}$ & $\mathbf{1 2 , 8 4 5 , 7 1 3}$ & $\mathbf{2 2 , 2 6 2 , 4 2 6}$ & $\mathbf{2 8 , 8 7 7 , 8 2 1}$ & $\mathbf{2 2 , 2 2 3 , 1 9 4}$ \\
\hline
\end{tabular}




\section{Horizontal and Vertical Analysis for the Period 2015-2016}

5.1 Horizontal Analysis for the Period 2015-2016

5.1.1 Horizontal Balance Sheet Analysis

Table 2. Horizontal analysis of the balance sheet for the period 2015-2016

\begin{tabular}{|c|c|c|c|c|c|}
\hline \multicolumn{6}{|l|}{ Balance Sheet } \\
\hline Balance Sheet Items & Counting & Year 2015 & Year 2016 & Sum e rr/z & $\%$ e rr/z \\
\hline \multicolumn{6}{|l|}{ Wealth (Active Bilacore) } \\
\hline \multicolumn{6}{|l|}{ Current assets } \\
\hline Cash and cash equivalents & 1 & $13,716,517$ & $17,544,076$ & $3,827,559$ & 27.9 \\
\hline Short-term financial instruments & 2 & $10,764,546$ & $16,243,056$ & $5,478,510$ & 50.89 \\
\hline Available-for-sale financial assets & 3 & 612,426 & $1,175,309$ & 562,883 & 91.91 \\
\hline $\mathrm{R} / \mathrm{A}$ & 4 & $22,549,741$ & $24,903,927$ & $2,354,186$ & 10.44 \\
\hline Advances & 5 & $1,340,947$ & $1,563,279$ & 222,332 & 16.58 \\
\hline Prepaid expenses & 6 & $2,174,832$ & $2,112,066$ & $-62,766$ & -2.89 \\
\hline Inventory & 7 & $14,673,434$ & $16,569,333$ & $1,895,899$ & 12.92 \\
\hline Other current assets & 8 & 923,288 & $1,365,023$ & 441,735 & 47.84 \\
\hline Total current assets & 9 & $66,755,731$ & $81,476,069$ & $14,720,338$ & 22.05 \\
\hline \multicolumn{6}{|l|}{ Non-current assets } \\
\hline Available-for-sale financial assets & 10 & $3,009,614$ & $4,882,060$ & $1,872,446$ & 62.22 \\
\hline Investments in other enterprises (j.v.) & 11 & $8,593,193$ & $8,202,305$ & $-390,888$ & -4.55 \\
\hline Land, factories and equipment & 12 & $57,925,451$ & $63,938,701$ & $6,013,250$ & 10.38 \\
\hline Intangible assets & 13 & $3,132,514$ & $3,482,126$ & 349,612 & 11.16 \\
\hline deposits & 14 & 739,299 & 760,613 & 21,314 & 2.88 \\
\hline Prepayment of long-term expenses & 15 & $3,224,914$ & $3,282,120$ & 57,206 & 1.77 \\
\hline Income tax prepayment (profit) & 16 & $1,664,724$ & $2,349,062$ & 684,338 & 41.11 \\
\hline Other fixed assets & 17 & 412,746 & 678,919 & 266,173 & 64.49 \\
\hline Total non-current assets & 18 & $78,702,455$ & $87,575,906$ & $8,873,451$ & 11.27 \\
\hline TOTAL PROPERTY & 19 & $145,458,186$ & $169,051,975$ & $23,593,789$ & 13.96 \\
\hline \multicolumn{6}{|l|}{ Liabilities and Capital } \\
\hline \multicolumn{6}{|l|}{ Short-term liabilities } \\
\hline ACCOUNTS PAYABLE & 20 & $17,280,823$ & $15,768,229$ & $-1,512,594$ & -8.75 \\
\hline Short-term loans & 21 & $9,012,904$ & $7,883,253$ & $-1,129,651$ & -12.53 \\
\hline Unearned income & 22 & $1,354,433$ & $1,416,928$ & 62,495 & 4.61 \\
\hline Withholding tax & 23 & $1,601,223$ & 902,226 & $-698,997$ & -43.65 \\
\hline Accrual costs & 24 & $7,304,386$ & $8,864,864$ & $1,560,478$ & 21.36 \\
\hline Profit tax payable & 25 & $1,178,973$ & $3,008,994$ & $1,830,021$ & 155.22 \\
\hline Current installment of long-term loans & 26 & 28,281 & 932,695 & 904,414 & 3197.96 \\
\hline Provisions & 27 & $3,281,240$ & $4,719,310$ & $1,438,070$ & 43.83 \\
\hline Other short-term liabilities & 28 & 334,839 & 321,120 & $-13,719$ & -4.1 \\
\hline Total current liabilities & 29 & $41,377,102$ & $43,817,619$ & $2,440,517$ & 5.9 \\
\hline Long-term liabilities & & & & & \\
\hline
\end{tabular}




\begin{tabular}{|l|r|r|r|r|r|} 
PAYABLE LIABILITIES & $\mathbf{3 1}$ & 956,777 & $1,088,489$ & 131,712 & 13.77 \\
\hline Long-term loans & $\mathbf{3 2}$ & $1,195,149$ & $1,707,940$ & 512,791 & 42.91 \\
\hline Other long-term liabilities & $\mathbf{3 3}$ & $3,438,028$ & $3,382,530$ & $-55,498$ & -1.61 \\
\hline Pension liability & $\mathbf{3 4}$ & $1,044,896$ & $1,615,105$ & 570,209 & 54.57 \\
\hline Profit tax (income) payable & $\mathbf{3 5}$ & $2,178,547$ & $3,201,818$ & $1,023,271$ & 46.97 \\
\hline Provisions & $\mathbf{3 6}$ & 339,112 & 381,411 & 42,299 & 12.47 \\
\hline Other long-term liabilities & & 340,180 & 440,757 & 100,577 & 29.57 \\
\hline Total long-term liabilities & $\mathbf{3 7}$ & $\mathbf{9 , 4 9 2 , 6 8 9}$ & $\mathbf{1 1 , 8 1 8 , 0 5 0}$ & $\mathbf{2 , 3 2 5 , 3 6 1}$ & $\mathbf{2 4 . 5}$ \\
\hline TOTAL LIABILITIES & $\mathbf{3 8}$ & $\mathbf{5 0 , 8 6 9 , 7 9 1}$ & $\mathbf{5 5 , 6 3 5 , 6 6 9}$ & $\mathbf{4 , 7 6 5 , 8 7 8}$ & $\mathbf{9 . 3 7}$ \\
\hline Equity & & & & & \\
\hline Preferential shares & $\mathbf{3 9}$ & 111,537 & 111,537 & & 0 \\
\hline Ordinary shares & $\mathbf{4 0}$ & 726,400 & 726,400 & 0 & 0 \\
\hline Premium from shares & $\mathbf{4 1}$ & $4,111,561$ & $4,111,561$ & 0 & 0 \\
\hline Retained earnings & $\mathbf{4 2}$ & $91,142,630$ & $112,020,996$ & $20,878,366$ & 22.91 \\
\hline Other reserves & $\mathbf{4 3}$ & $-5,446,639$ & $-7,649,185$ & $-2,202,546$ & 40.44 \\
\hline Uncontrolled interest & $\mathbf{4 4}$ & $3,942,906$ & $4,094,997$ & 152,091 & 3.86 \\
\hline TOTAL EQUITY & $\mathbf{4 5}$ & $\mathbf{9 4 , 5 8 8 , 3 9 5}$ & $\mathbf{1 1 3 , 4 1 6 , 3 0 6}$ & $\mathbf{1 8 , 8 2 7 , 9 1 1}$ & $\mathbf{1 9 . 9 1}$ \\
\hline Total liabilities and capital & $\mathbf{4 6}$ & $\mathbf{1 4 5 , 4 5 8 , 1 8 6}$ & $\mathbf{1 6 9 , 0 5 1 , 9 7 5}$ & $\mathbf{2 3 , 5 9 3 , 7 8 9}$ & $\mathbf{1 6 . 2 2}$ \\
\hline
\end{tabular}

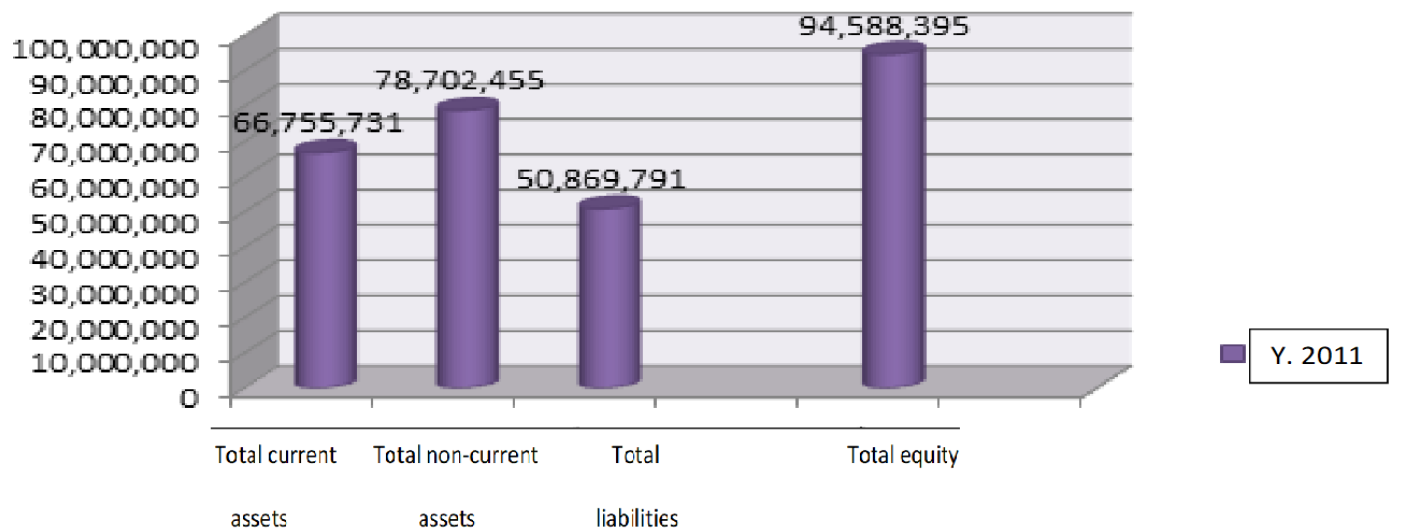

Chart 3. Balance sheet in 2015

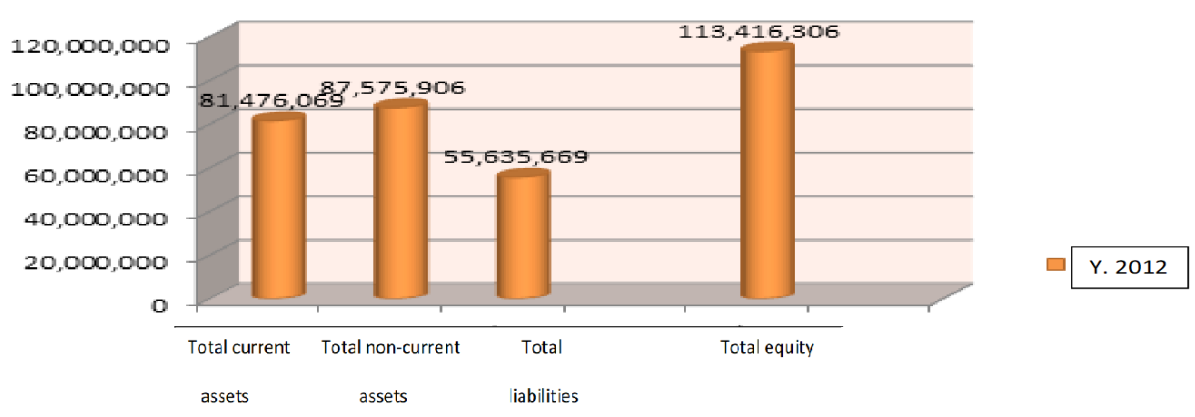

Chart 4. Balance sheet in 2016 


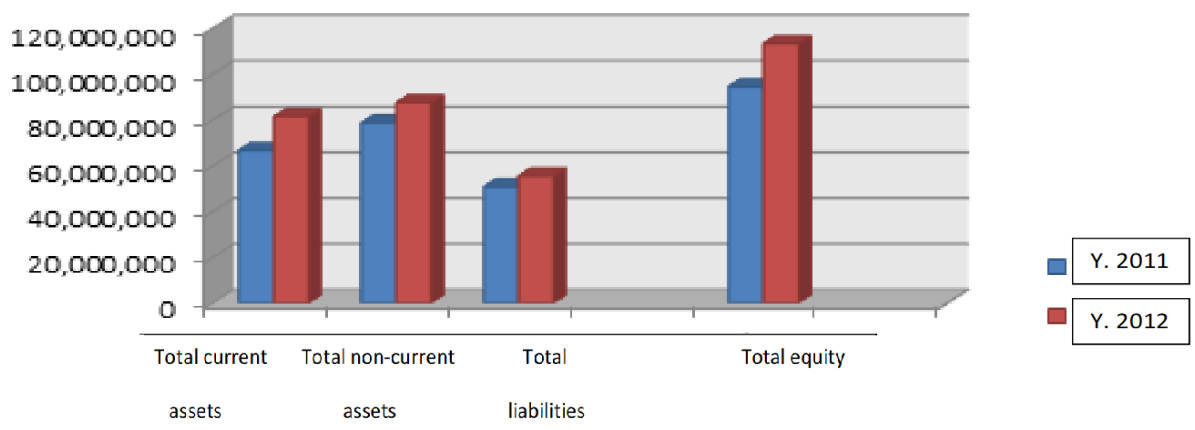

Chart 5. Balance sheet in 2015 and 2016

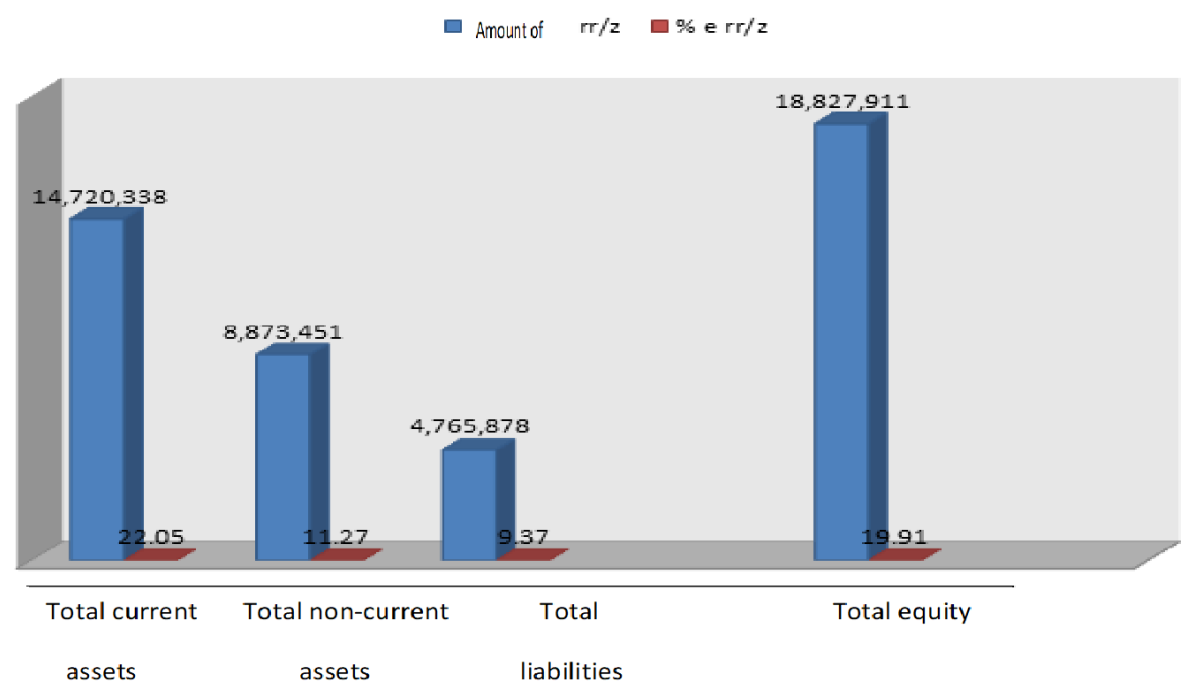

Chart 6. Horizontal analysis of the balance sheet for the years 2015-2016

\subsubsection{Horizontal Analysis of the Income and Expenditure Statement}

Table 3. Horizontal analysis of the income and expenditure statement for the years 2015-2016

\begin{tabular}{llllll}
\hline Income and Expenditure Statement & \multicolumn{5}{l}{} \\
\hline ITEMS & Counting & Year 2015 & Year 2016 & Amount of rr/z & \% of rr/z \\
\hline Revenues from shooting & $\mathbf{4 7}$ & $\mathbf{1 5 4 , 0 4 8 , 8 9 5}$ & $\mathbf{1 8 7 , 7 5 4 , 2 8 3}$ & $\mathbf{3 3 , 7 0 5 , 3 8 8}$ & $\mathbf{2 1 . 8 8}$ \\
Cost of goods sold & $\mathbf{4 8}$ & $(104,700,887)$ & $(118,244,730)$ & $(13,543,843)$ & 12.94 \\
Gross Profit & $\mathbf{4 9}$ & $\mathbf{4 9 , 3 4 8 , 0 0 8}$ & $\mathbf{6 9 , 5 0 9 , 5 5 3}$ & $20,161,545$ & 40.86 \\
Operating expenses & $\mathbf{5 0}$ & $(34,742,191)$ & $(42,388,520)$ & $(7,646,329)$ & 22.01 \\
Operating Profit & $\mathbf{5 1}$ & $\mathbf{1 4 , 6 0 5 , 8 1 7}$ & $\mathbf{2 7 , 1 2 1 , 0 3 3}$ & $\mathbf{1 2 , 5 1 5 , 2 1 6}$ & $\mathbf{8 5 . 6 9}$ \\
Financial income & $\mathbf{5 2}$ & $1,444,895$ & 808,215 & $(636,680)$ & $(44.06)$ \\
Profit before tax & $\mathbf{5 3}$ & $\mathbf{1 6 , 0 5 0 , 7 1 2}$ & $\mathbf{2 7 , 9 2 9 , 2 4 8}$ & $\mathbf{1 1 , 8 7 8 , 5 3 6}$ & $\mathbf{7 4 . 0 1}$ \\
Income tax expense & $\mathbf{5 4}$ & $\mathbf{( 3 , 2 0 4 , 9 9 9 )}$ & $(5,666,822)$ & $(2,461,823)$ & 76.81 \\
Profit of the year & $\mathbf{5 5}$ & $\mathbf{1 2 , 8 4 5 , 7 1 3}$ & $\mathbf{2 2 , 2 6 2 , 4 2 6}$ & $\mathbf{9 , 4 1 6 , 7 1 3}$ & $\mathbf{0 . 0 0}$ \\
\hline
\end{tabular}

\subsection{Vertical Analysis for the Period 2015-2016}

5.2.1 Vertical Balance Sheet Analysis

Table 4. Vertical analysis of the balance sheet for the years 2015-2016

\begin{tabular}{|l|r|r|r|r|c|}
\hline Balance Sheet & Counting & Year 2015 & Year 2016 & Amount of rr/z & $\%$ of rr/z \\
\hline Balance Sheet Items & Cous
\end{tabular}




\begin{tabular}{|c|c|c|c|c|c|}
\hline \multicolumn{6}{|l|}{ Wealth (Active Balance) } \\
\hline \multicolumn{6}{|l|}{ Current assets } \\
\hline Cash and cash equivalents & 1 & $13,716,517$ & $17,544,076$ & $3,827,559$ & 27.9 \\
\hline Short-term financial instruments & 2 & $10,764,546$ & $16,243,056$ & $5,478,510$ & 50.89 \\
\hline Available-for-sale financial assets & 3 & 612,426 & $1,175,309$ & 562,883 & 91.91 \\
\hline $\mathrm{R} / \mathrm{A}$ & 4 & $22,549,741$ & $24,903,927$ & $2,354,186$ & 10.44 \\
\hline Advances & 5 & $1,340,947$ & $1,563,279$ & 222,332 & 16.58 \\
\hline Prepaid expenses & 6 & $2,174,832$ & $2,112,066$ & $-62,766$ & -2.89 \\
\hline Inventory & 7 & $14,673,434$ & $16,569,333$ & $1,895,899$ & 12.92 \\
\hline Other current assets & 8 & 923,288 & $1,365,023$ & 441,735 & 47.84 \\
\hline Total current assets & 9 & $66,755,731$ & $81,476,069$ & $14,720,338$ & 22.05 \\
\hline \multicolumn{6}{|l|}{ Non-current assets } \\
\hline Available-for-sale financial assets & 10 & $3,009,614$ & $4,882,060$ & $1,872,446$ & 62.22 \\
\hline Investments in other enterprises (j.v.) & 11 & $8,593,193$ & $8,202,305$ & $-390,888$ & -4.55 \\
\hline Land, factories and equipment & 12 & $57,925,451$ & $63,938,701$ & $6,013,250$ & 10.38 \\
\hline Intangible assets & 13 & $3,132,514$ & $3,482,126$ & 349,612 & 11.16 \\
\hline deposits & 14 & 739,299 & 760,613 & 21,314 & 2.88 \\
\hline Prepayment of long-term expenses & 15 & $3,224,914$ & $3,282,120$ & 57,206 & 1.77 \\
\hline Income tax prepayment (profit) & 16 & $1,664,724$ & $2,349,062$ & 684,338 & 41.11 \\
\hline Other fixed assets & 17 & 412,746 & 678,919 & 266,173 & 64.49 \\
\hline Total non-current assets & 18 & $\mathbf{7 8 , 7 0 2 , 4 5 5}$ & $87,575,906$ & $8,873,451$ & 11.27 \\
\hline TOTAL PROPERTY & 19 & $145,458,186$ & $169,051,975$ & $23,593,789$ & 13.96 \\
\hline \multicolumn{6}{|l|}{ Liabilities and Capital } \\
\hline \multicolumn{6}{|l|}{ Short-term liabilities } \\
\hline ACCOUNTS PAYABLE & 20 & $17,280,823$ & $15,768,229$ & $-1,512,594$ & -8.75 \\
\hline Short-term loans & 21 & $9,012,904$ & $7,883,253$ & $-1,129,651$ & -12.53 \\
\hline Unearned income & 22 & $1,354,433$ & $1,416,928$ & 62,495 & 4.61 \\
\hline Withholding tax & 23 & $1,601,223$ & 902,226 & $-698,997$ & -43.65 \\
\hline Accrual costs & 24 & $7,304,386$ & $8,864,864$ & $1,560,478$ & 21.36 \\
\hline Profit tax payable & 25 & $1,178,973$ & $3,008,994$ & $1,830,021$ & 155.22 \\
\hline Current installment of long-term loans & 26 & 28,281 & 932,695 & 904,414 & 3197.96 \\
\hline Provisions & 27 & $3,281,240$ & $4,719,310$ & $1,438,070$ & 43.83 \\
\hline Other short-term liabilities & 28 & 334,839 & 321,120 & $-13,719$ & -4.1 \\
\hline Total current liabilities & 29 & $41,377,102$ & $43,817,619$ & $2,440,517$ & 5.9 \\
\hline \multicolumn{6}{|l|}{ Long-term liabilities } \\
\hline PAYABLE LIABILITIES & 31 & 956,777 & $1,088,489$ & 131,712 & 13.77 \\
\hline Long-term loans & 32 & $1,195,149$ & $1,707,940$ & 512,791 & 42.91 \\
\hline Other long-term liabilities & 33 & $3,438,028$ & $3,382,530$ & $-55,498$ & -1.61 \\
\hline Pension liability & 34 & $1,044,896$ & $1,615,105$ & 570,209 & 54.57 \\
\hline Profit tax (income) payable & 35 & $2,178,547$ & $3,201,818$ & $1,023,271$ & 46.97 \\
\hline Provisions & 36 & 339,112 & 381,411 & 42,299 & 12.47 \\
\hline Other long-term liabilities & & 340,180 & 440,757 & 100,577 & 29.57 \\
\hline Total long-term liabilities & 37 & $9,492,689$ & $11,818,050$ & $2,325,361$ & 24.5 \\
\hline TOTAL LIABILITIES & 38 & $50,869,791$ & $55,635,669$ & $4,765,878$ & 9.37 \\
\hline \multicolumn{6}{|l|}{ Equity } \\
\hline Preferential shares & 39 & 111,537 & 111,537 & 0 & 0 \\
\hline Ordinary shares & 40 & 726,400 & 726,400 & 0 & 0 \\
\hline Premium from shares & 41 & $4,111,561$ & $4,111,561$ & 0 & 0 \\
\hline Retained earnings & 42 & $91,142,630$ & $112,020,996$ & $20,878,366$ & 22.91 \\
\hline Other reserves & 43 & $-5,446,639$ & $-7,649,185$ & $-2,202,546$ & 40.44 \\
\hline Uncontrolled interest & 44 & $3,942,906$ & $4,094,997$ & 152,091 & 3.86 \\
\hline TOTAL EQUITY & 45 & $\mathbf{9 4 , 5 8 8 , 3 9 5}$ & $113,416,306$ & $18,827,911$ & 19.91 \\
\hline Total liabilities and capital & 46 & $145,458,186$ & $169,051,975$ & $23,593,789$ & 16.22 \\
\hline
\end{tabular}




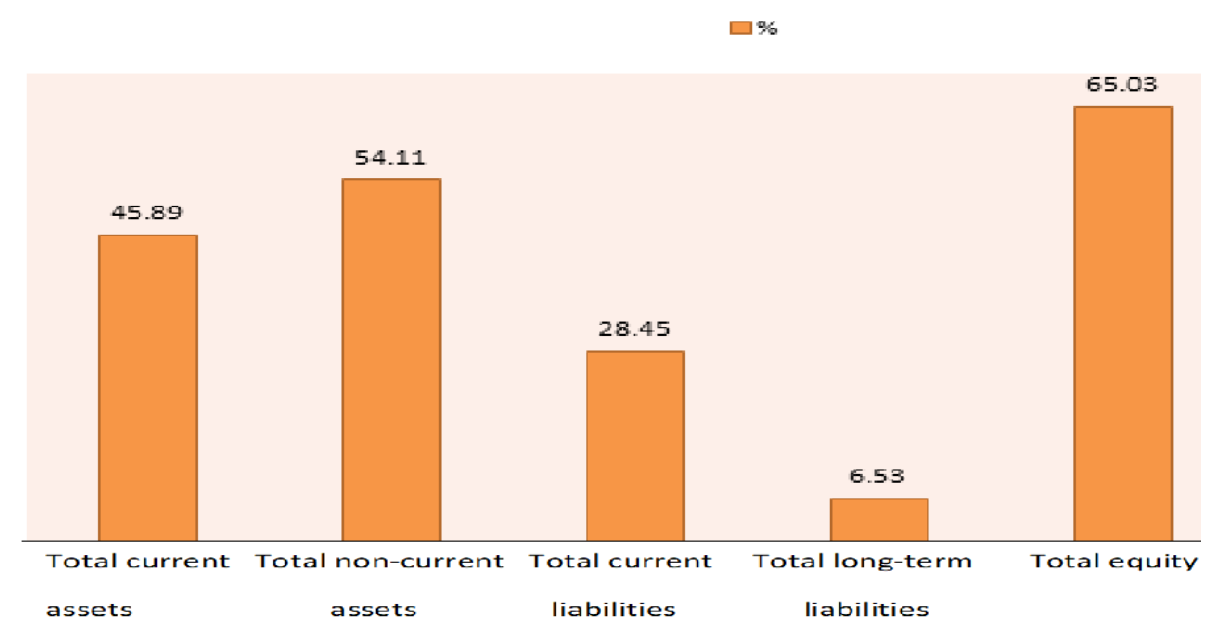

Chart 9. Percentage of growth of assets, liabilities and capital 2015

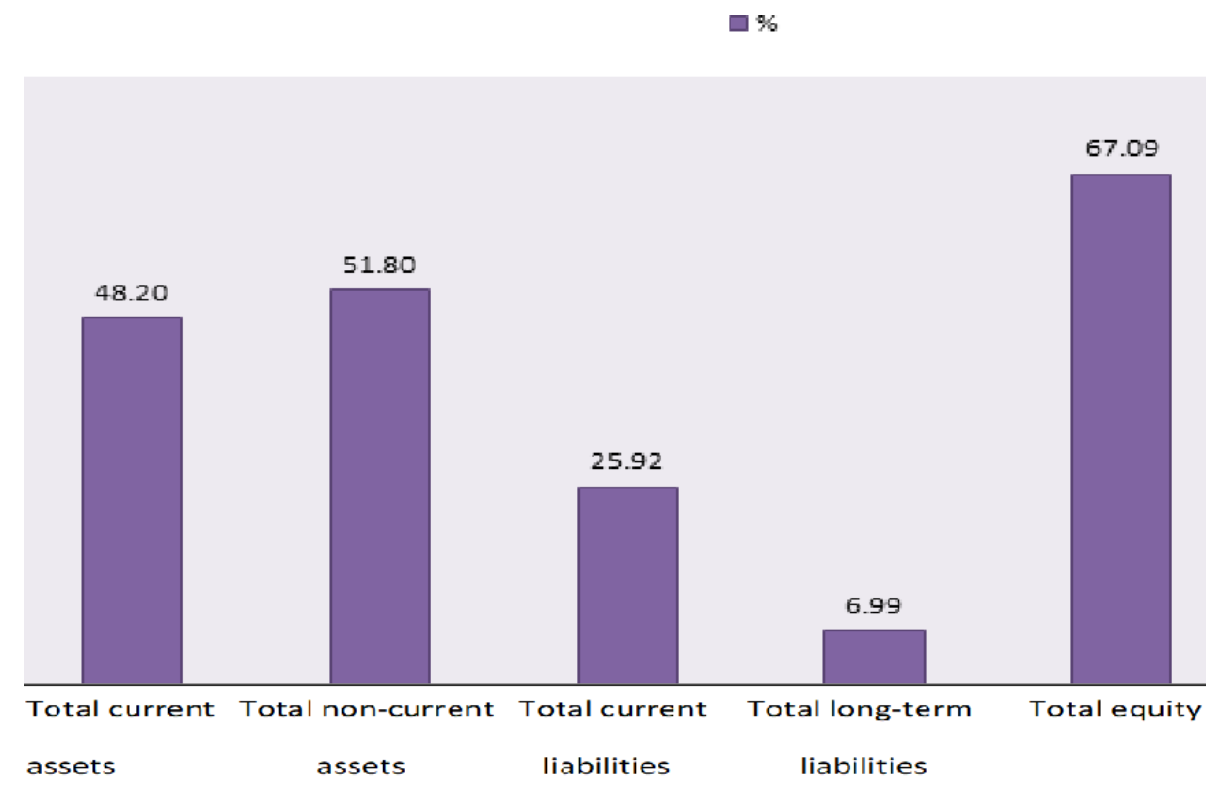

Chart 10. Percentage growth of assets, liabilities and capital 2016

\subsubsection{Vertical Analysis of SIE}

Table 5. Vertical and horizontal analysis of the income statement for the years 2015-2016

\begin{tabular}{llll}
\hline Income statement & Counting & $\mathbf{\%}$ & \\
\hline Voices & 1 & $\mathbf{1 0 0 . 0 0}$ & $\mathbf{1 0 0}$ \\
\hline Revenues from sales & 2 & $(67.97)$ & $(62.98)$ \\
Cost of goods sold & 3 & $\mathbf{3 2 . 0 3}$ & $\mathbf{3 7 . 0 2}$ \\
Gross Profit & 4 & $(22.55)$ & $(22.58)$ \\
Operating expenses & 5 & $\mathbf{9 . 4 8}$ & $\mathbf{1 4 . 4 4}$ \\
Operating Profit & 6 & 0.94 & 0.43 \\
Financial income & 7 & $\mathbf{1 0 . 4 2}$ & $\mathbf{1 4 . 8 8}$ \\
Profit before tax & 8 & $(2.08)$ & $(3.02)$ \\
Income tax expense & 9 & $\mathbf{8 . 3 4}$ & $\mathbf{1 1 . 8 6}$ \\
Profit of the year & & & \\
\hline
\end{tabular}




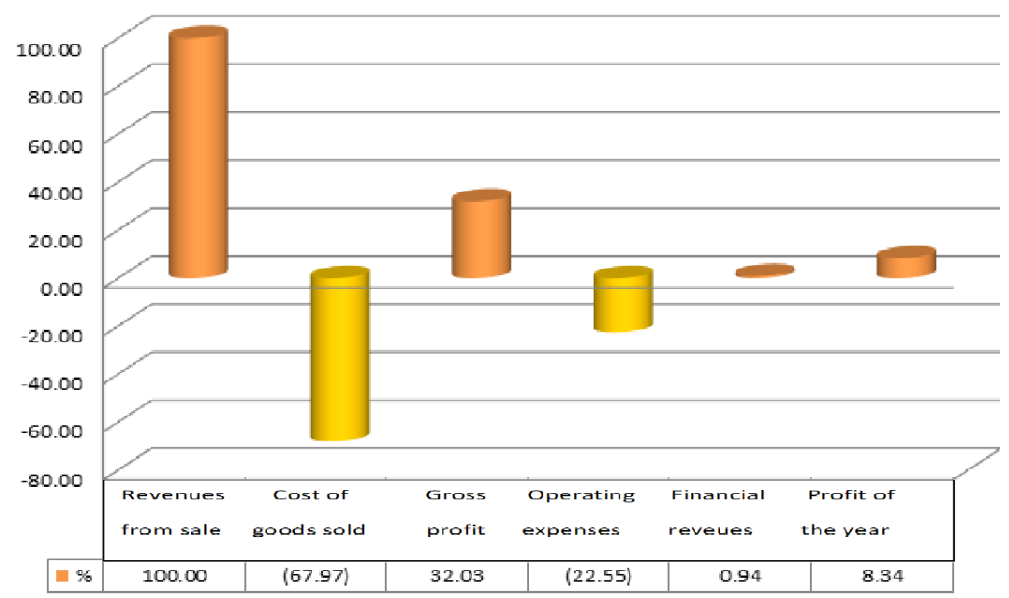

Chart 11. Vertical and horizontal analysis of the income and expenditure statement for 2015

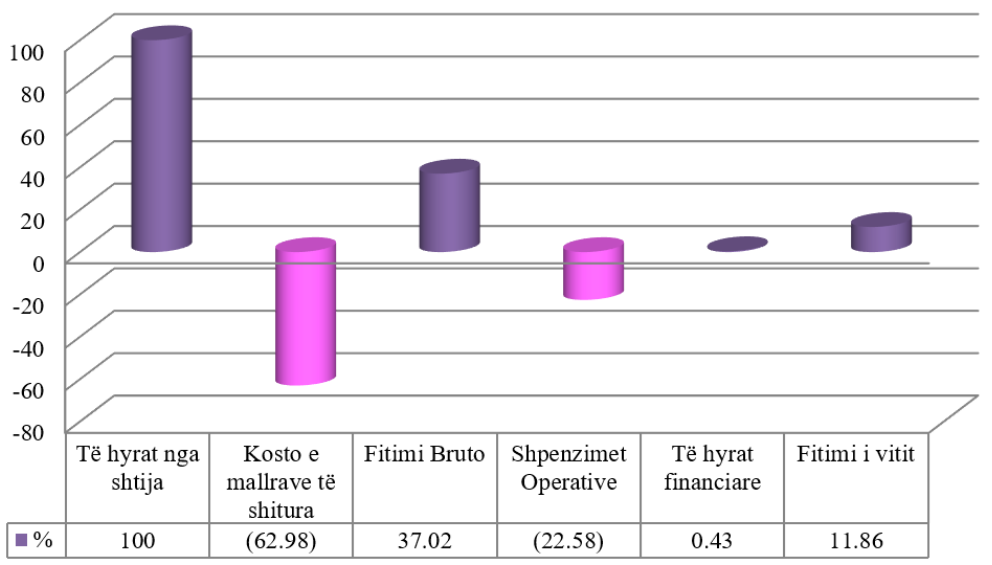

Chart 12. Vertical and horizontal analysis of the income and expenditure statement for 2016

\section{Horizontal and Vertical Analysis for the Period 2017-2018}

6.1 Horizontal Analysis for the Period 2017-2018

6.1.1 Horizontal Balance Sheet Analysis

Table 6. Horizontal analysis of the balance sheet for the period 2017-2018

\begin{tabular}{llrrrr}
\hline Balance sheet & & & & \\
Balance Sheet Items & Counting & Year 2017 & Year 2018 & Sum of the rr/z & \%ofrr/z \\
\hline Assets & & & & & \\
\hline Current assets & $\mathbf{1}$ & $15,431,422$ & $15,997,688$ & 566,266 & 3.67 \\
\hline Money and its equivalents & $\mathbf{2}$ & $34,798,353$ & $39,602,713$ & $4,804,360$ & 13.81 \\
Short-term financial instruments & $\mathbf{3}$ & $1,410,525$ & $3,122,255$ & $1,711,730$ & 121.35 \\
Available-for-sale financial assets & $\mathbf{3}$ & $26,415,175$ & $26,821,018$ & $(405,843)$ & $(1.54)$ \\
Accounts receivable & $\mathbf{4}$ & $1,827,147$ & $1,889,874$ & 62,727 & 3.43 \\
Advances & $\mathbf{5}$ & $2,343,362$ & $3,179,057$ & 835,695 & 35.66 \\
Prepaid expenses & $\mathbf{6}$ & $18,132,160$ & $16,450,560$ & $(1,681,600)$ & $(9.27)$ \\
Inventory & $\mathbf{7}$ & $2,574,370$ & 613,177 & $(1,961,193)$ & $(76.18)$ \\
Assets held for sale & $\mathbf{8}$ & $2,023,680$ & $1,705,275$ & $(318,405)$ & $(12.37)$ \\
Other current assets & $\mathbf{9}$ & $\mathbf{1 0 4 , 9 5 6 , 1 9 4}$ & $\mathbf{1 0 9 , 3 8 1 , 6 1 7}$ & $\mathbf{4 , 4 2 5 , 4 2 3}$ & $\mathbf{4 . 2 2}$ \\
Total current assets & $\mathbf{1 0}$ & & & & \\
Non-current assets & & & & & \\
\hline
\end{tabular}




\begin{tabular}{|c|c|c|c|c|c|}
\hline Available-for-sale financial assets & 11 & $5,911,475$ & $12,033,351$ & $6,121,876$ & 103.56 \\
\hline Investments in other enterprises & 12 & $6,085,750$ & $4,970,515$ & $(1,115,235)$ & $(18.33)$ \\
\hline Land, factories and equipment & 13 & $71,540,214$ & $76,824,309$ & $5,284,095$ & 7.39 \\
\hline Intangible assets & 14 & $3,772,008$ & $4,545,904$ & 773,896 & 20.52 \\
\hline Prepayment of long-term expenses & 15 & $3,284,168$ & $4,613,970$ & $1,329,802$ & 40.49 \\
\hline Income tax prepayment (profit) & 16 & $4,379,589$ & $4,299,986$ & $(79,603)$ & $(1.82)$ \\
\hline Other fixed assets & 17 & $2,927,627$ & $2,217,933$ & $(709,694)$ & $(24.24)$ \\
\hline Total non-current assets & 18 & $97,900,831$ & $109,505,968$ & $11,605,137$ & 11.85 \\
\hline Total assets & 19 & $202,857,025$ & 218,887,585 & $16,030,560$ & 7.90 \\
\hline \multicolumn{6}{|c|}{ Detyrimet dhe Kapitali Liabilities and Capital } \\
\hline Accounts payable & 20 & $16,709,661$ & $7,518,480$ & $(9,191,181)$ & $(55.01)$ \\
\hline Short-term loans & 21 & $6,101,125$ & $7,627,338$ & $1,526,213$ & 25.02 \\
\hline Advances received & 22 & $1,616,899$ & $1,355,780$ & $(261,119)$ & $(16.15)$ \\
\hline Withholding taxes & 23 & $1,114,419$ & $1,103,482$ & $(10,937)$ & $(0.98)$ \\
\hline Accrual costs & 24 & $10,750,052$ & $12,232,143$ & $1,482,091$ & 13.79 \\
\hline Profit tax payable & 25 & $3,208,583$ & $2,052,920$ & $(1,155,663)$ & $(36.02)$ \\
\hline Loans and bonds & 26 & $2,298,712$ & $1,689,624$ & $(609,088)$ & $(26.50)$ \\
\hline Provisions & 27 & $6,383,470$ & $5,691,565$ & $(691,905)$ & $(10.84)$ \\
\hline Liabilities held for sale & 28 & & 26,898 & 26,898 & \\
\hline Other short-term liabilities & 29 & 443,450 & $10,111,780$ & $9,668,330$ & 2180.25 \\
\hline Total current liabilities & 30 & $48,626,371$ & $49,410,010$ & 783,639 & 1.61 \\
\hline \multicolumn{6}{|l|}{ Long-term liabilities } \\
\hline Unsecured bonds & 31 & $1,242,365$ & $1,288,004$ & 45,639 & 3.67 \\
\hline Long-term loans & 32 & 933,495 & 96,581 & $(836,914)$ & $(89.65)$ \\
\hline Liabilities payable & 33 & 998,537 & $2,433,999$ & $1,435,462$ & 143.76 \\
\hline Net defined benefit obligations & 34 & $1,757,701$ & 191,262 & $(1,566,439)$ & $(89.12)$ \\
\hline Deferred income tax liabilities & 35 & $5,697,310$ & $3,892,667$ & $(1,804,643)$ & $(31.68)$ \\
\hline Provisions & 36 & 436,771 & 474,295 & 37,524 & 8.59 \\
\hline Other long-term liabilities & 37 & $1,009,628$ & $1,427,368$ & 417,740 & 41.38 \\
\hline Total liabilities & 38 & $60,702,178$ & $59,214,186$ & $(1,487,992)$ & $(2.45)$ \\
\hline \multicolumn{6}{|l|}{ Equity } \\
\hline Preferential shares & 39 & 113,207 & 113,486 & 279 & 0.25 \\
\hline Ordinary shares & 40 & 737,276 & 739,097 & 1,821 & 0.25 \\
\hline Premium from shares & 41 & $4,173,119$ & $4,183,426$ & 10,307 & 0.25 \\
\hline Retained earnings & 42 & $140,813,307$ & $161,042,656$ & $20,229,349$ & 14.37 \\
\hline Other reserves & 43 & $(8,963,398)$ & $(12,092,132)$ & $(3128734)$ & 34.91 \\
\hline Other comprehensive income & 44 & & 76,091 & 76,091 & \\
\hline Uncontrolled interest & 45 & $5,281,336$ & $5,610,775$ & 329,439 & 6.24 \\
\hline Total Equity & 46 & $142,154,847$ & $159,673,399$ & $\mathbf{1 7 , 5 1 8 , 5 5 2}$ & 12.32 \\
\hline Total liabilities and capital & 47 & $202,857,025$ & $218,887,585$ & $16,030,560$ & 7.90 \\
\hline
\end{tabular}

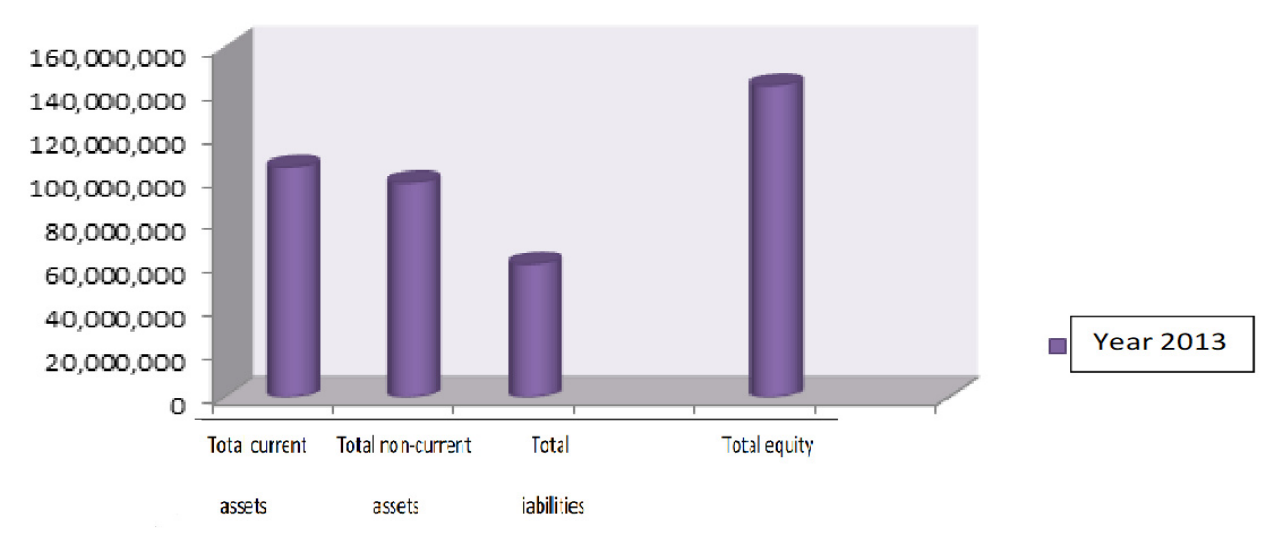

Chart 13. Balance sheet in 2017 

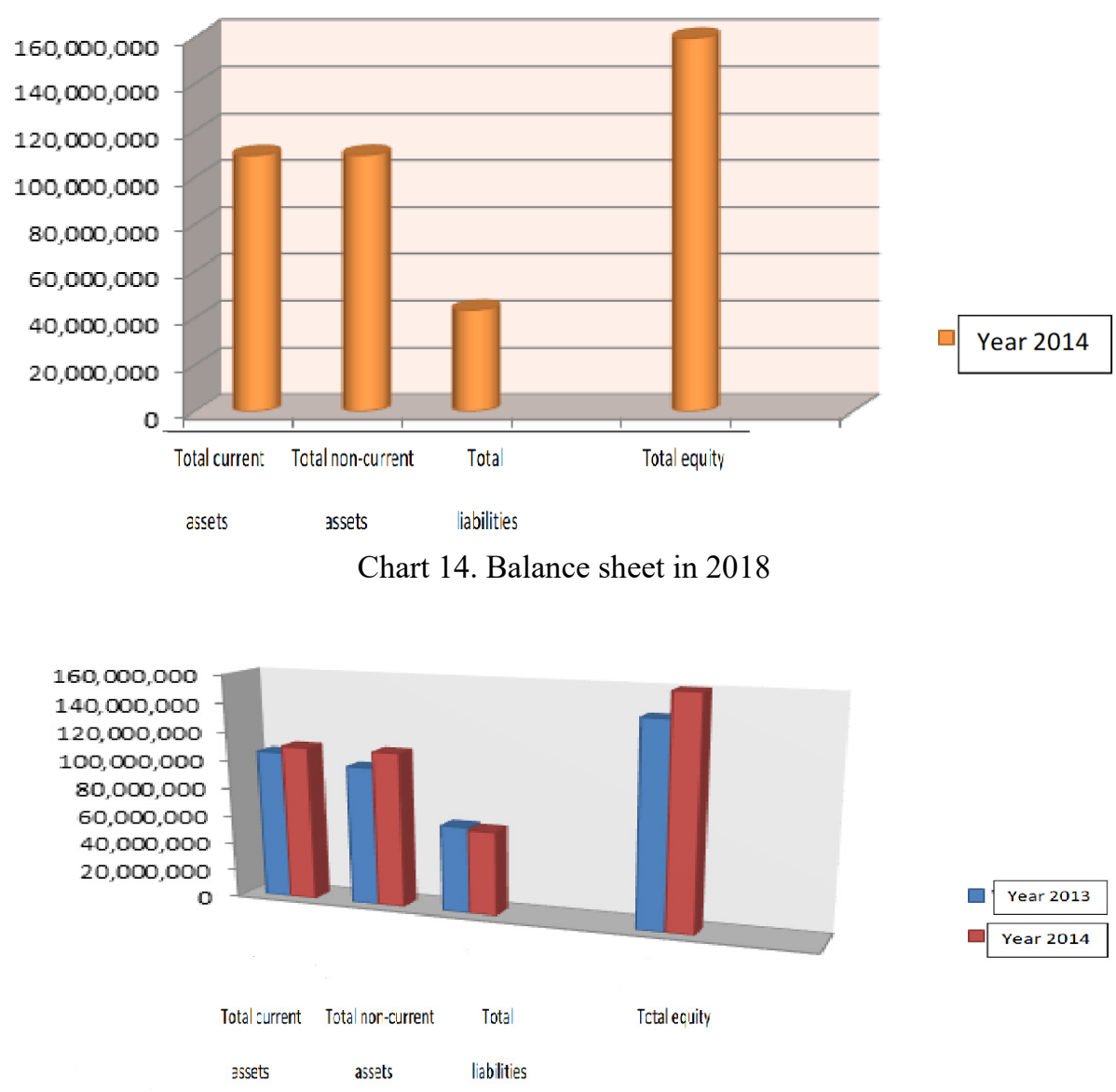

Chart 15. Balance sheet in 2017 and 2018

6.1.2 Horizontal Analysis of SIE

Table 7. Vertical and horizontal analysis of the income and expenditure statement for the years 2017-2018

\begin{tabular}{lcrrrr}
\hline Income and Expenditure Statement & & & & \\
\hline Voices & Counting & Year 2017 & Year 2018 & Amount of rr/z & \% of rr/z \\
Revenues from sales & $\mathbf{4 8}$ & $\mathbf{2 1 6 7 0 8 6 7 7}$ & $\mathbf{1 9 5 8 8 2 9 5 5}$ & $\mathbf{( 2 0 8 2 5 7 2 2 )}$ & $\mathbf{( 9 . 6 1 )}$ \\
Cost of goods sold & $\mathbf{4 9}$ & $(130480725)$ & $(121856939)$ & 8623786 & $(6.61)$ \\
Gross Profit & $\mathbf{5 0}$ & $\mathbf{8 6 2 2 7 9 5 2}$ & $\mathbf{7 4 0 2 6 0 1 6}$ & $\mathbf{( 1 2 2 0 1 9 3 6 )}$ & $\mathbf{( 1 4 . 1 5 )}$ \\
Operating expenses & $\mathbf{5 1}$ & $(51370553)$ & $(50253744)$ & 1116809 & $(2.17)$ \\
Operating Profit & $\mathbf{5 2}$ & $\mathbf{3 4 8 5 7 3 9 9}$ & $\mathbf{2 3 7 7 2 2 7 2}$ & $\mathbf{( 1 1 0 8 5 1 2 7 )}$ & $\mathbf{( 3 1 . 8 0 )}$ \\
Financial income & $\mathbf{5 3}$ & 1469509 & 2707288 & 1237779 & 84.23 \\
Profit before tax & $\mathbf{5 4}$ & $\mathbf{3 6 3 5 3 9 0 8}$ & $\mathbf{2 6 4 7 9 5 6 0}$ & $\mathbf{( 9 8 7 4 3 4 8 )}$ & $\mathbf{( 2 7 . 1 6 )}$ \\
Income tax expense & $\mathbf{5 5}$ & $\mathbf{( 7 4 7 6 0 8 7 )}$ & $(4256366)$ & 3219721 & $(43.07)$ \\
Profit of the year & $\mathbf{5 6}$ & $\mathbf{2 8 8 7 7 8 2 1}$ & $\mathbf{2 2 2 2 3 1 9 4}$ & $\mathbf{( 6 6 5 4 6 2 7 )}$ & $\mathbf{( 2 3 . 0 4 )}$ \\
\hline
\end{tabular}




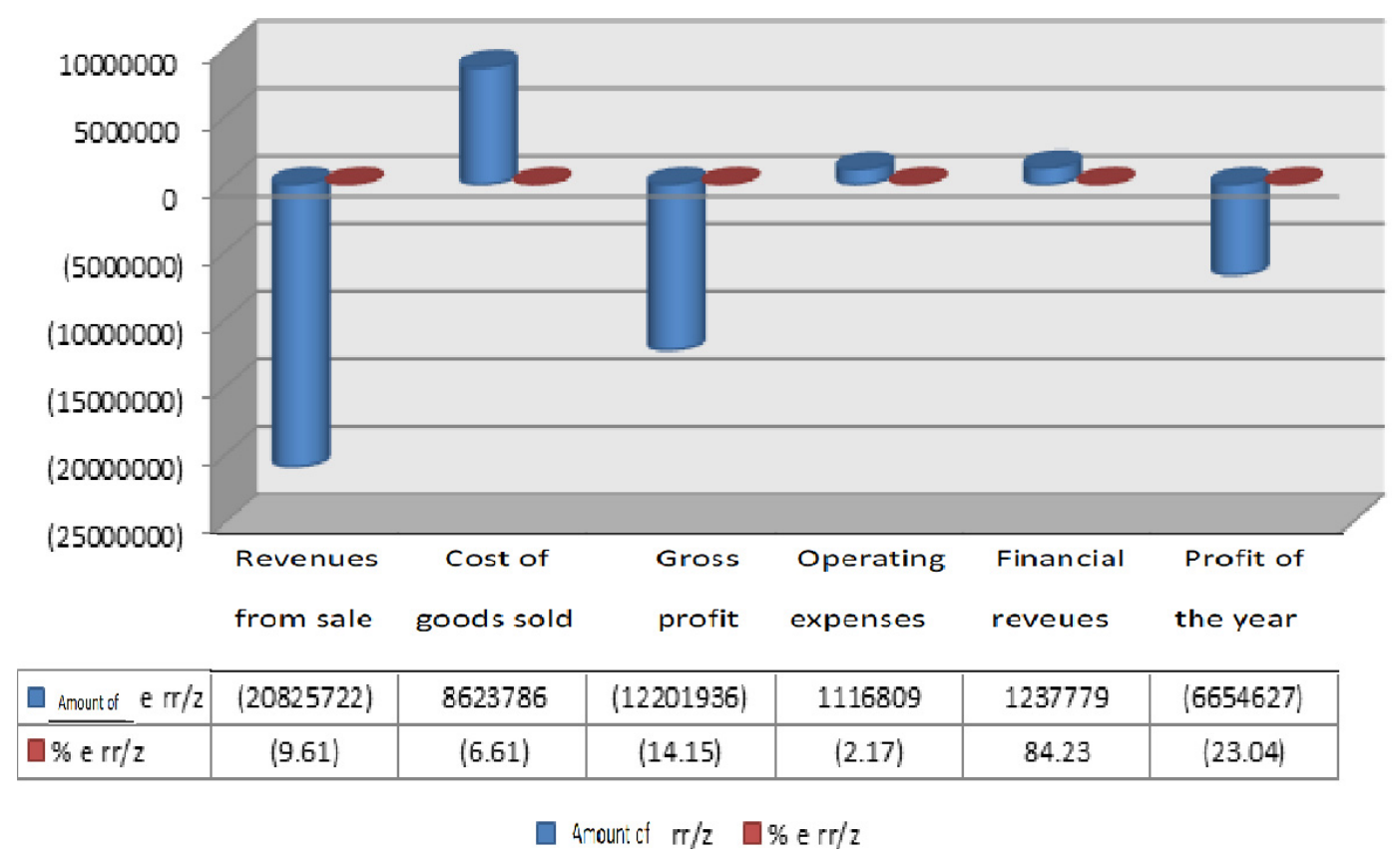

Chart 18. Horizontal analysis of the income and expenditure statement for the years 2017-2018

\subsection{Vertical Analysis for the Period 2017-2018}

6.2.1 Vertical Balance Sheet Analysis

Table 8. Vertical analysis of the balance sheet for the years 2017-2018

\begin{tabular}{llll}
\hline Balance Sheet & & & \\
\hline Balance Sheet Items & Counting & \% & \% \\
\hline Assets (Balance Sheet Assets) & & & \\
\hline Current assets & $\mathbf{1}$ & 7.61 & 7.31 \\
Money and its equivalents & $\mathbf{2}$ & 17.15 & 18.09 \\
Short-term financial instruments & $\mathbf{3}$ & 0.70 & 1.43 \\
Available-for-sale financial assets & $\mathbf{4}$ & 13.02 & 12.25 \\
Accounts receivable & $\mathbf{5}$ & 0.90 & 0.86 \\
Advances & $\mathbf{6}$ & 1.16 & 1.45 \\
Prepaid expenses & $\mathbf{7}$ & 8.94 & 7.52 \\
Inventory & $\mathbf{8}$ & 1.27 & 0.28 \\
Assets held for sale & $\mathbf{9}$ & 1.00 & 0.78 \\
Other current assets & $\mathbf{1 0}$ & 51.74 & 49.97 \\
Total current assets & & & \\
Non-current assets & $\mathbf{1 1}$ & 2.91 & 5.50 \\
Available-for-sale financial assets & $\mathbf{1 2}$ & 3.00 & 2.27 \\
Investments in other enterprises & $\mathbf{1 3}$ & 35.27 & 35.10 \\
Land, factories and equipment & $\mathbf{1 4}$ & 1.86 & 2.08 \\
Intangible assets & $\mathbf{1 5}$ & 1.62 & 2.11 \\
Prepayment of long-term expenses & $\mathbf{1 6}$ & 2.16 & 1.96 \\
Income tax prepayment (profit) & $\mathbf{1 7}$ & 1.44 & 1.01 \\
Other fixed assets & $\mathbf{1 8}$ & 48.26 & 50.03 \\
Total non-current assets & $\mathbf{1 9}$ & $\mathbf{1 0 0 . 0 0}$ & $\mathbf{1 0 0 . 0 0}$ \\
\hline Total assets & & & \\
\hline Liabilities and Equity (Balance Sheet Liabilities) & & & 3.43 \\
Short-term liabilities & $\mathbf{2 0}$ & 8.24 & 3.48 \\
Accounts payable & $\mathbf{2 1}$ & 3.01 & \\
Short-term loans & & & \\
& & & \\
\end{tabular}


Advances received

Withholding taxes

Accrual costs

Profit tax payable

Loans and bonds

Provisions

Liabilities held for sale

Other short-term liabilities

Total current liabilities

Long-term liabilities

Unsecured bonds

Long-term loans

Liabilities payable

Net defined benefit obligations

Deferred income tax liabilities

Provisions

Other long-term liabilities

Total long-term liabilities

Total liabilities

Equity

Preferential shares

Ordinary shares

Premium from shares

Retained earnings

Other reserves

Other comprehensive income held for sale

Uncontrolled interest

Total Equity

Total liabilities and capital
$22 \quad 0.80 \quad 0.62$

$23 \quad 0.55 \quad 0.50$

$\begin{array}{lll}24 & 5.30 & 5.59\end{array}$

$25 \quad 1.58 \quad 0.94$

$26 \quad 1.13 \quad 0.77$

$\begin{array}{lll}27 & 3.15 & 2.60\end{array}$

$28 \quad 0.01$

$29 \quad 0.22 \quad 4.62$

$\begin{array}{lll}30 & 23.97 & 22.57\end{array}$

$\begin{array}{lll}31 & 0.61 & 0.59\end{array}$

$32 \quad 0.46 \quad 0.04$

$33 \quad 0.49 \quad 1.11$

$\begin{array}{lll}34 & 0.87 & 0.09\end{array}$

$35 \quad 2.81 \quad 1.78$

$36 \quad 0.22 \quad 0.22$

$\begin{array}{lll}37 & 0.50 & 0.65\end{array}$

$38 \quad 5.95 \quad 4.48$

$392 \quad 29.92 \quad 27.05$

$40 \quad 0.06 \quad 0.05$

$41 \quad 0.36 \quad 0.34$

$42 \quad 2.06 \quad 1.91$

$\begin{array}{lll}43 & 69.42 & 73.57\end{array}$

$44 \quad(4.42) \quad(5.52)$

$45 \quad 0.03$

$46 \quad 2.60 \quad 2.56$

$47 \quad \mathbf{7 0 . 0 8} \quad \mathbf{7 2 . 9 5}$

$48 \quad 100.00 \quad 100.00$

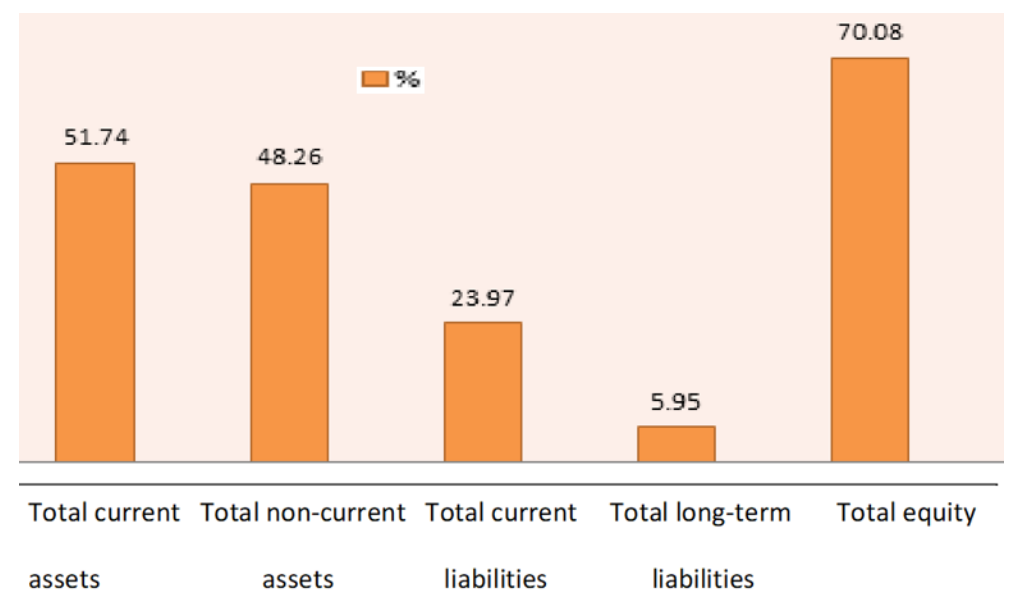

Chart 19. Percentage of assets, liabilities and capital growth 2017 


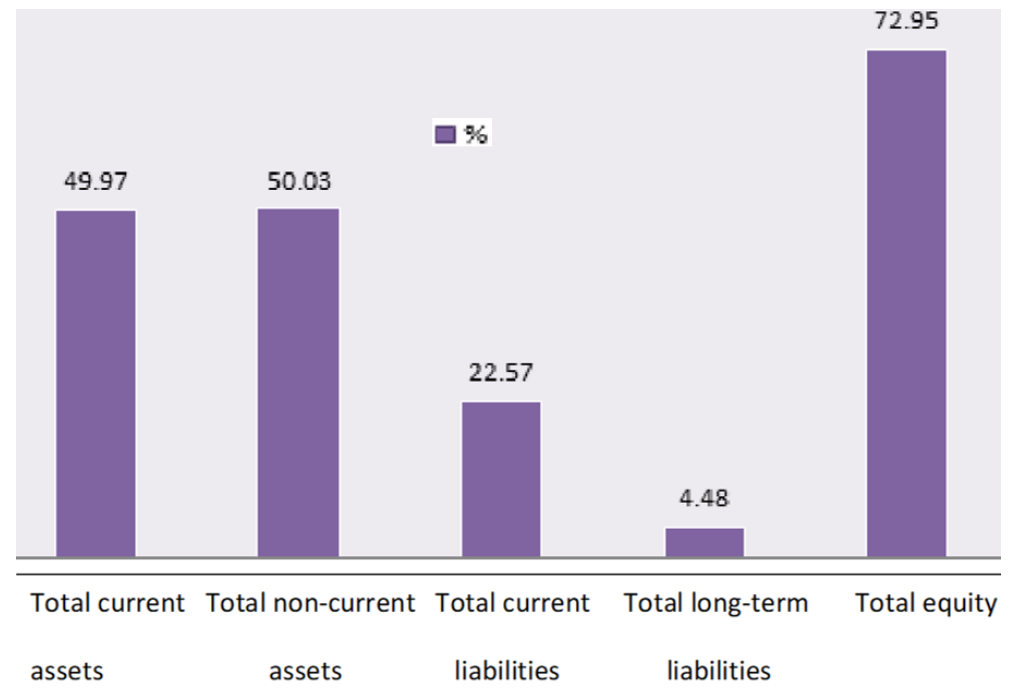

Chart 20. Percentage increase in assets, liabilities and capital 2018

\subsubsection{Vertical Analysis of the Income Statement}

Table 9. Vertical analysis of the income statement for the years 2017-2018

\begin{tabular}{llll}
\hline Income and Expenditure Statement & & & \\
\hline Voices & Counting & \% & \% \\
Revenues from sales & $\mathbf{4 9}$ & $\mathbf{1 0 0 . 0 0}$ & $\mathbf{1 0 0 . 0 0}$ \\
Cost of goods sold & $\mathbf{5 0}$ & $(60.21)$ & $(62.21)$ \\
Gross Profit & $\mathbf{5 1}$ & $\mathbf{3 9 . 7 9}$ & $\mathbf{3 7 . 7 9}$ \\
Operating expenses & $\mathbf{5 2}$ & $(23.70)$ & $(25.65)$ \\
Operating Profit & $\mathbf{5 3}$ & $\mathbf{1 6 . 0 8}$ & $\mathbf{1 2 . 1 4}$ \\
Financial income & $\mathbf{5 4}$ & 0.68 & 1.38 \\
Profit before tax & $\mathbf{5 5}$ & $\mathbf{1 6 . 7 8}$ & $\mathbf{1 3 . 5 2}$ \\
Income tax expense & $\mathbf{5 6}$ & $(3.45)$ & $(2.17)$ \\
Profit of the year & $\mathbf{5 7}$ & $\mathbf{1 3 . 3 3}$ & $\mathbf{1 1 . 3 5}$ \\
\hline
\end{tabular}

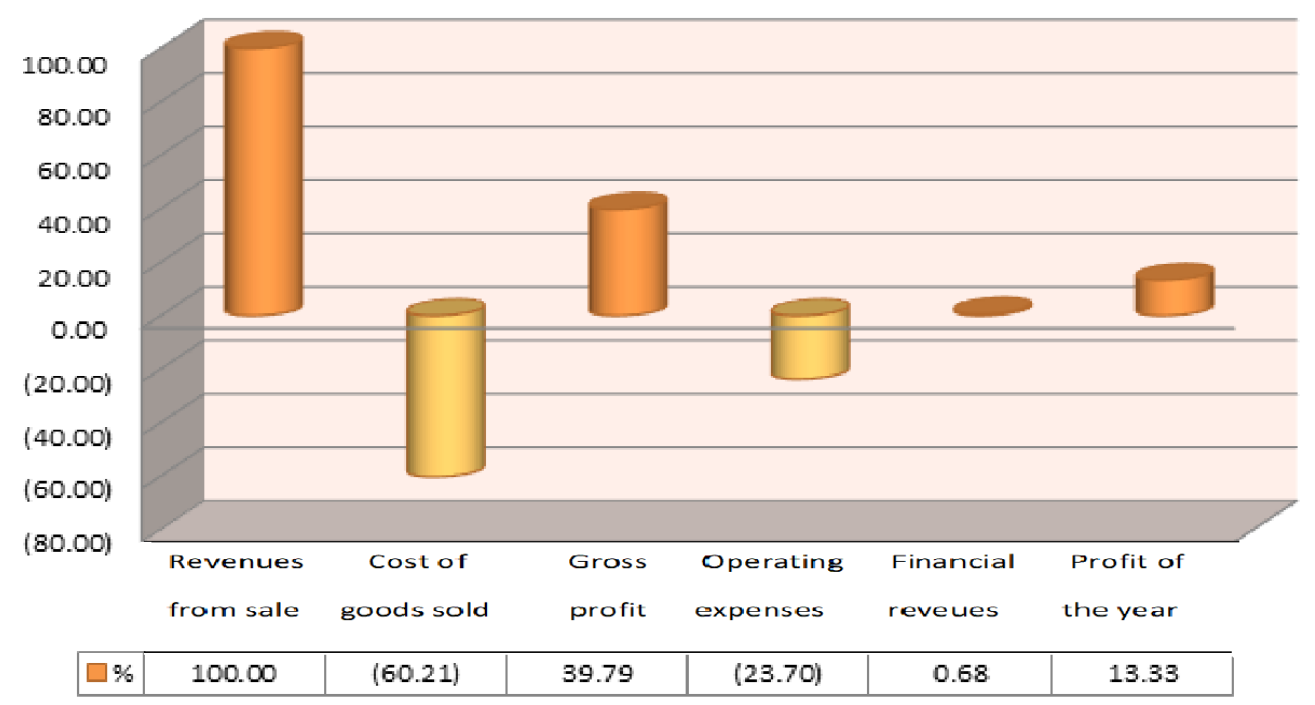

Chart 21. Vertical analysis of the income and expenditure statement for 2017 


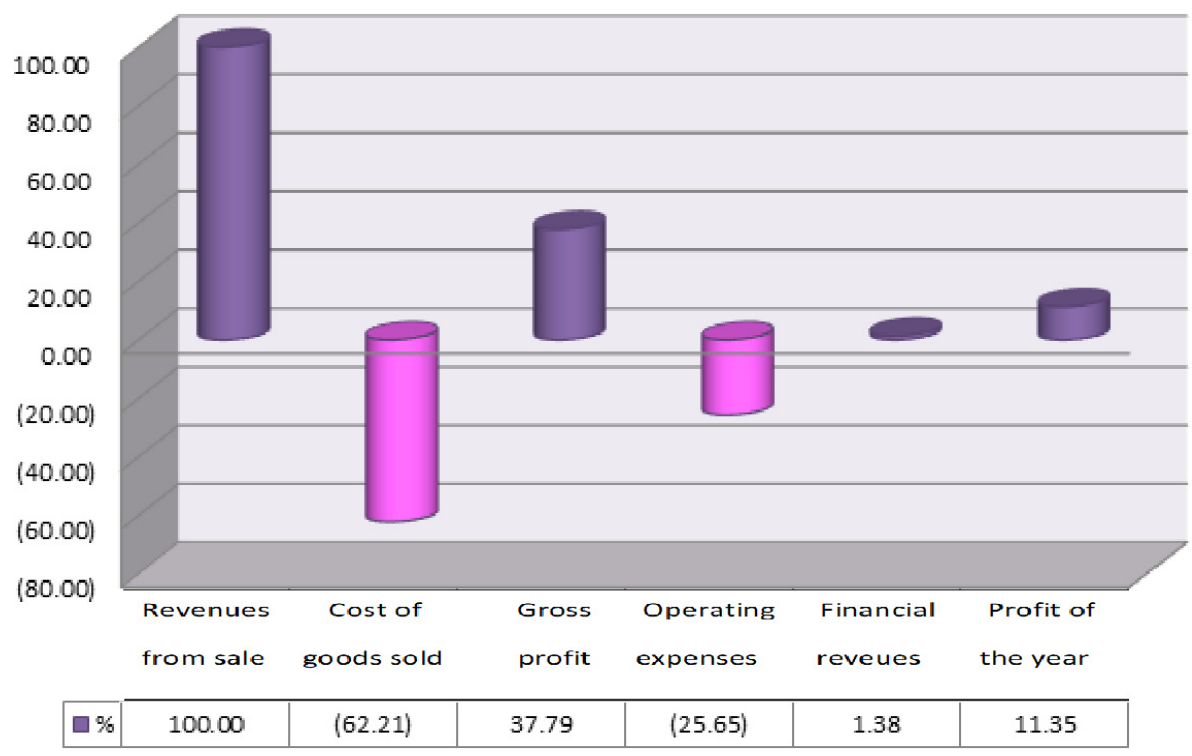

Chart 22. Vertical analysis of the income and expenditure statement for 2018

\section{Commenting of the Results from the Analysis for the Year 2015 - 2018}

\subsection{Conclusions for Horizontal Analysis}

From the analysis made for the business of "Samsung" we notice that 2016 was more successful than 2015, through these analyzes we conclude that wealth in 2016 increased by $€ 23,593,789$ or $13.96 \%$ compared to 2015 , respectively current assets increased by $€ 14,720,338$ or $22.05 \%$ and non-current assets $€ 8,873,451$ or $11.27 \%$ similarly occurred with liabilities, it increased by $€ 23,593,789$ or $16.22 \%$, liabilities increased by $€ 4,765,878$ or 9.37\% including short-term liabilities in the amount of $€ 2,440,517$ or $5.90 \%$ and long-term ones by $€ 2,325,361$ or $24.50 \%$, even equity has increased in the amount of $€ 18,827,911$ or $19.91 \%$.

We note that financial assets available for sale is the item that has experienced the most increase, specifically $91.91 \%$, followed by other fixed assets by $64.49 \%$, short-term financial instruments by $50.89 \%$ and so on, so we can conclude that "Samsung" has invested in different forms the profit of last year but also that a part of these investments are financed from external sources since we have increased liabilities, during 2016 they have increased by $€ 4,765,878$ or $9.37 \%$, namely short-term liabilities are increased by $€ 2,440,517$ or $5.90 \%$ and long-term liabilities by $€ 2,325,361$ or $24.50 \%$, withholding tax is the item that has decreased the most by $43.65 \%$ but we have an enormous increase of profit tax payable by $155.22 \%$, ie the company despite the fact that it does not endanger liquidity has not fulfilled its obligations. Within equity, we have an increase of $19.91 \%$ or $€ 18,827,9911$, during this year "Samsung" has not issued any regular or ordinary shares, while the increase of the retained profit by $22.91 \%$ is noticea

Revenues from sales in 2016 were higher than in 2015 by $€ 33,705,388$ or $21.88 \%$, generally all expenses were higher, KMSH increased by $12.94 \%$, operating expenses by $22.01 \%$ while financial revenues decreased by $44.06 \%$. The net profit in 2016 is higher compared to 2015 by $€ 9,416,716$ or $73.31 \%$.

From the analysis of the years 2017-2018 we notice that assets in 2018 increased by $€ 16,030,560$ or $7.90 \%$, within it current assets increased by $€ 4,425,423$ or $4.22 \%$ and non-current assets by $€ 11,605,137$ or $11.85 \%$. Liabilities this year decreased by $€ 1,478,992$ or $2.45 \%$, respectively short-term liabilities increased by only $€ 783,639$ or $1.61 \%$ while equity increased by $€ 17,518,552$ or $12.32 \%$. Within the structure of assets, financial assets available for sale (short-term and long-term) are the items with the highest increase, and intangible assets have increased in 2018 compared to 2017 by $20.52 \%$, it is important to note that the inventory of goods is decreased by $9.27 \%$, maybe even "Samsung" is applying the method "Just In Time" or has controlled more production by selling from last year's stocks. In terms of liabilities, long-term loans have decreased the most by $89.65 \%$, short-term accounts have decreased by $55.01 \%$, generally during this year the company has significantly fulfilled liabilities to partners, financing is mainly done from internal sources, during this year "Samsung" has issued regular and preferred shares at the same percentage of $0.25 \%$, retained earnings for this period increased by $14.37 \%$. 
Revenues from sales for the accounting period 2018 are lower by $€ 20,825,722$ or $9.61 \%$ compared to 2017 , the cost of goods sold together with operating expenses have decreased by $6.61 \%$ and $2.17 \%$ respectively, financial revenues have increased for $84.23 \%$, while the net profit is lower for $6,654,6277$ or $23.04 \%$.

\subsection{Conclusions for Vertical Analysis}

Based on the results of the vertical analysis of the years 2015-2016 we see that current assets in 2016 participate in total assets with $48.20 \%$ while in 2015 with $45.89 \%$, current assets in 2016 participate in total $51.80 \%$ and in 2015 with $54.11 \%$, in this structure land, factory and equipment has the largest share in both periods $39.82 \%$ (2015) and $37.82 \%$ (2016), followed by accounts receivable $15.50 \%$ (2015) and $14.73 \%(2016)$ this item carries the potential risk of turning into bad debt in case of non-collection, while money participates with $9.43 \%$ (2015) respectively with $10.38 \%$ (2016). Liabilities have a share of $32.91 \%$ in the total liabilities for 2016 , while for 2015 with $34.97 \%$. During these two years, the participation within the structure of liabilities does not have much movement, for 2015 short-term liabilities participate with $28.45 \%$ and long-term liabilities with $6.53 \%$, while in 2016 with $25.92 \%$ respectively $6.99 \%$. If we take into account all items of liabilities, accounts payablehave the largest share with $11.88 \%$ (2015) and $9.33 \%$ (2016). The equity share in the balance sheet liability is $65.03 \%$ (2015) and $67.09 \%$ (2016). Within the equity category, the items of preferred and regular shares do not differ much if we compare their participation in both periods for the fact that the enterprise during this period did not issue any type of share. Preferred shares participate $0.8 \%$ (2015) and $0.7 \%$ (2016), while ordinary shares $0.50 \%(2015)$ and $0.43 \%$ (2016). Slightly larger change is observed in retained earnings which in the total liabilities for 2015 participates $62.66 \%$, while $66.26 \%$ for 2016 .

In terms of income statement, the cost of goods sold participates in sales revenues $67.97 \%$ in 2015 and $62.98 \%$ in 2016, while gross profit $32.03 \%$ participates in sales revenues for 2015 , slightly different there is also 2016 where the share of gross profit in sales revenues is $37.02 \%$. Operating expenses have almost the same share in both years $22.55 \%$ (2015) and $22.58 \%$ (2016), operating profit for 2015 participates $9.48 \%$ in sales revenues, while $14.44 \%$ in 2016 . Of total revenues $11.86 \%$ remain net profit in 2016 while $8.34 \%$ in 2015 .

From the vertical analysis of 2017-2018 we see that the share of current and non-current assets in total assets is almost proportional, in 2017 the share of current assets is $51.74 \%$ and non-current assets $48.26 \%$, while in 2018 current assets participate $49.97 \%$ and non-circulating $50.03 \%$. Within this category, the largest share is occupied by land, factories and equipment with $35 \%$ in both periods, from this result we can conclude that the company has made investments in this item since it has not been reduced by depreciation but has increased compared to 2017 . Other items with higher participation are short-term financial instruments with participation $17.15 \%$ (2017) and $18.09 \%$ (2018), and accounts receivable with participation 13.02\% (2017) and $12.25 \%$ (2018) which carry the risk of turning into bad debt due to the possibility of non-collection. In the balance sheet liabilities participate with $29.92 \%$ in 2017 and $27.25 \%$ in 2018 , concretely short-term liabilities participate with $23.97 \%$ in 2017 and $22.57 \%$ in 2018, while long-term liabilities participate with 5.95\% in 2017 and $4.48 \%$ in 2018 . None of the liabilities items have a significant share, however what can be noticed is that "Samsung" has proved to be a trusted partner as it has largely paid the liabilities to accounts payable, this is evidenced by the percentage of participation, in 2017 accounts payablehad a share of $8.24 \%$ while in 2018 the share was $3.43 \%$. The equity share in the balance sheet liability for 2017 is $70.08 \%$ while in 2018 it is $72.95 \%$, the largest share within the equity is occupied by the retained profit with $69.42 \%$ in 2017 and $73.57 \%$ in 2018 .

From the analysis of the financial statement it results that the share of costs of goods sold is $60.21 \%$ in 2017 and $62.21 \%$ in 2018 , gross profit participates with $39.79 \%$ (2017) and $37.79 \%$ (2018), the share of operating expenses in it total revenue is $23.70 \%$ (2017) and $25.65 \%$ (2018). As it is noticed the difference of the percentage of items between the two periods varies by $2 \%$, the same happens with the net profit, in 2017 the share of net profit in sales revenue is $13.33 \%$ while in 2018 it is $11.35 \%$.

\section{Results Obtained from the Hypotheses Laid out}

\subsection{Testing the First Hypothesis}

Understanding investment growth as a potential growth of the overall enterprise represents the purpose of achieving this paper. The first hypothesis was that the increase in foreign investment, as Samsung did during the 2015-2018 period, will have a positive impact on the growth of the enterprise.

Thus from hypothesis testing we derive the null hypothesis and the alternative hypothesis. They are as follows:

H1o The growth of foreign investment as Samsung did during the period 2015-2018, will have a positive impact on the growth of entrepreneurship 
H1a: The increase in foreign investment, as Samsung did during the 2015-2018 period, will negatively affect the growth of the enterprise.

To test this hypothesis, Regression analysis was used, which results in:

Table 10. Regression analysis first hypothesis-Anova

\begin{tabular}{|c|c|c|c|c|c|c|c|}
\hline \multicolumn{8}{|c|}{ Model Summary ${ }^{b}$} \\
\hline \multirow[b]{3}{*}{ Model } & \multirow[b]{3}{*}{$\mathrm{R}$} & \multirow[b]{2}{*}{$\mathrm{R}$} & \multirow[b]{2}{*}{ Adjusted R } & \multirow{3}{*}{$\begin{array}{l}\text { Std. Error of the } \\
\text { Estimate }\end{array}$} & \multicolumn{3}{|c|}{ Change Statistics } \\
\hline & & & & & R Square & $\mathrm{F}$ & Sig. F \\
\hline & & Square & Square & & Change & Change df1 df2 & Change \\
\hline 1 & $.436^{\mathrm{a}}$ & .190 & .162 & .763 & .190 & 4115 & .000 \\
\hline
\end{tabular}

a. Predictors: (Constant), Availability of resources for specific departments of the enterprise, Achievement of the enterprise to arouse investment interest, Foreign investments and their distribution in the enterprise, Distribution of planned resources of potential investment

b. Dependent Variable:

Increased foreign investment and its impact on entrepreneurship

Table 11. Regression analysis first hypothesis-Anova

\begin{tabular}{lllllll}
\hline ANOVA $^{\mathbf{a}}$ & & & & & \\
\hline Model & & Sum of Squares & df & Mean Square & F & Sig. \\
1 & Regression & 15.719 & 4 & 3.930 & 6.758 & $.000^{\mathrm{b}}$ \\
& Residual & 66.872 & 115 & .581 & & \\
\hline & Total & 82.592 & 119 & & & \\
\hline
\end{tabular}

a. Dependent Variable:

Increased foreign investment and its impact on entrepreneurship

b. Predictors: (Constant), Availability of resources for specific departments of the enterprise, Achievement of the enterprise to arouse investment interest, Foreign investments and their distribution in the enterprise, Distribution of planned resources of potential investment

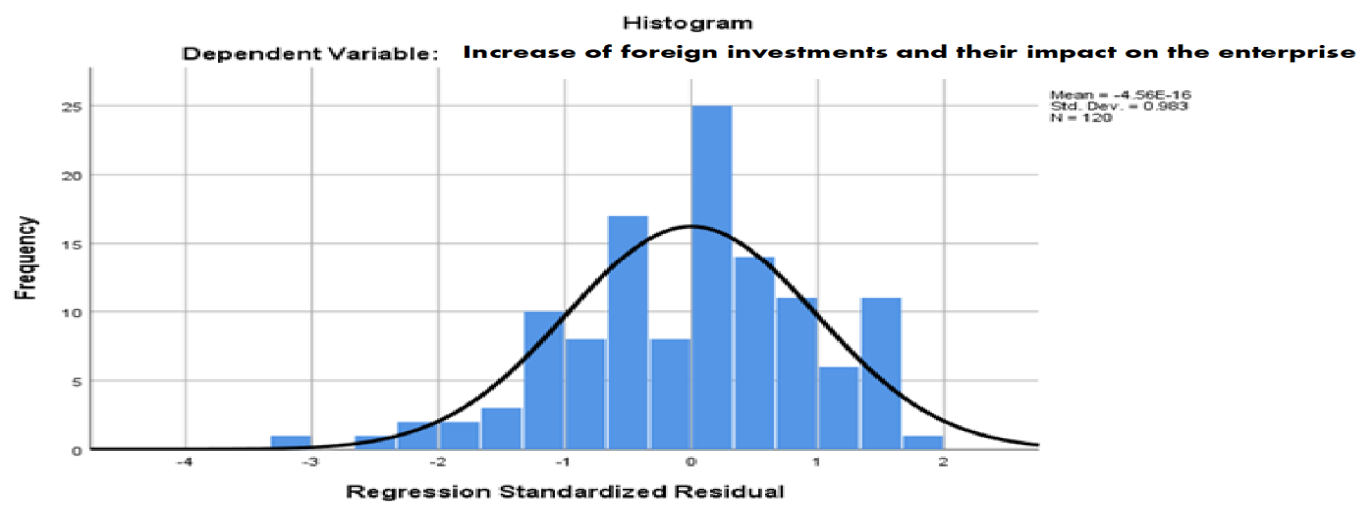

Chart 23. Regression analysis first hypothesis-Histogram 


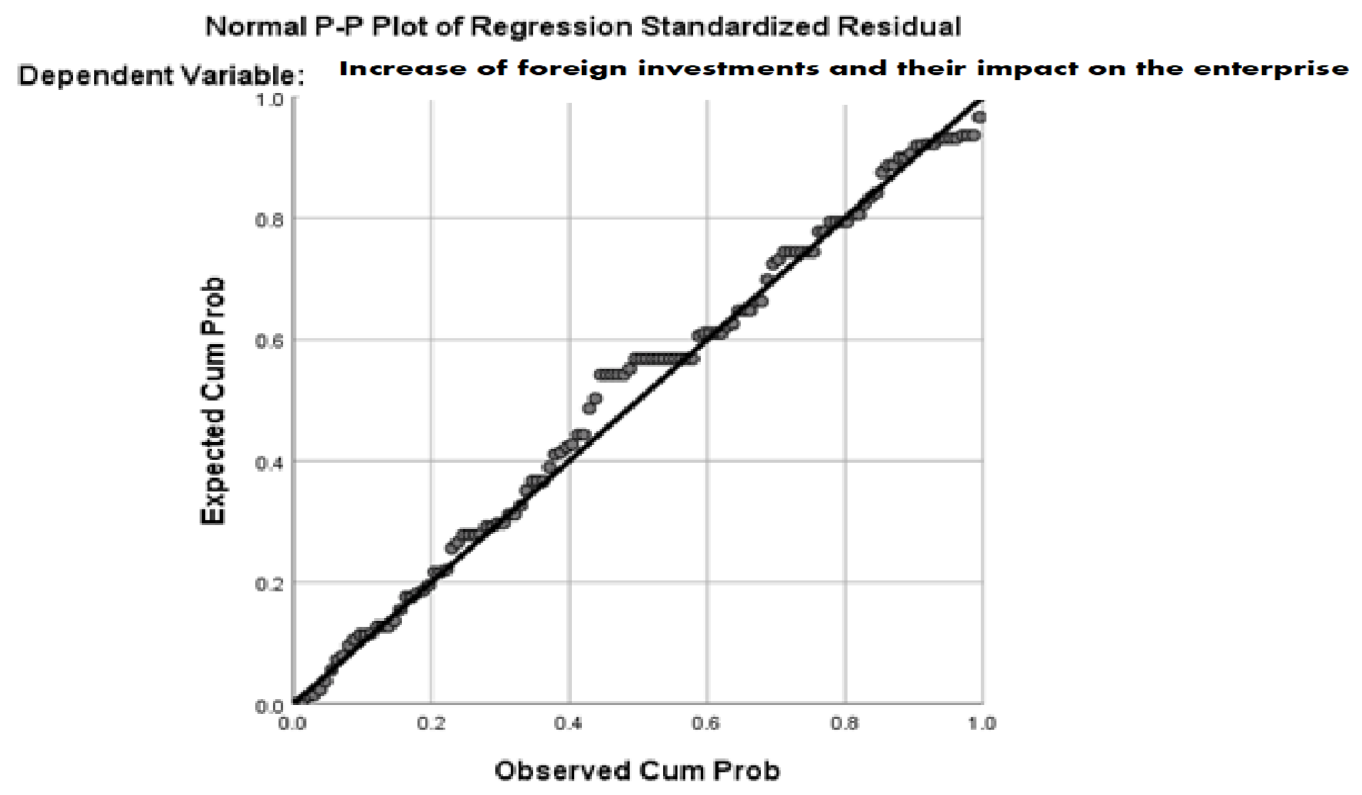

Chart 24. Regression analysis first hypothesis- Normal probability plot

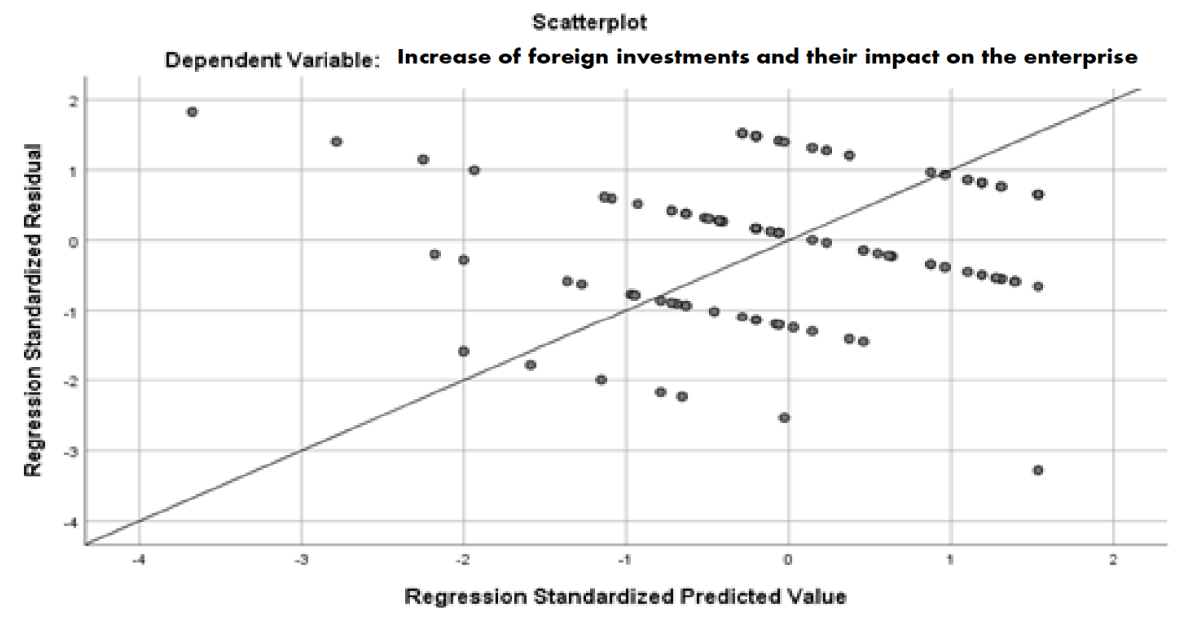

Chart 25. Regression analysis first hypothesis

For the first hypothesis the value of R Square turns out to be 0.190 and the significance level is 0 .

This makes us understand that the impact of foreign investment growth and its impact on entrepreneurship can be determined or predicted by $19.0 \%$.

So there is a big impact of workers' desire for investment. Independent variables such as the availability of resources for specific departments of the enterprise, the achievement of the enterprise to arouse investment interest, foreign investment and their distribution in the enterprise, the distribution of resources planned potential investment, mark a major impact. The value "Sig.000" confirms this result because $\mathrm{P}<0.05$.

So, Hypothesis H1o: The increase of foreign investments as Samsung did during the period 2015-2018, will have a positive impact on the growth of the enterprise, it is accepted according to the Program used SPSS and the model used: Regression. The null hypothesis is approved and the alternative hypothesis is rejected. 


\subsection{Testing of the Second Hypothesis}

\section{The Second Hypothesis}

Identifying how the increase in accounts receivable and the decrease in accounts payableby employees would affect the next goal of this paper. The second hypothesis was: Increasing accounts receivable and reducing accounts payablein the enterprise, would positively affect the enterprise

Thus from hypothesis testing we derive the null hypothesis and the alternative hypothesis. They are as follows:

H2o: Increasing accounts receivable and reducing accounts payablein the enterprise, would have a positive impact.

H2a: Increasing accounts receivable and reducing accounts payablein the enterprise would have a negative impact.

To test this hypothesis, Regression analysis was used, which results in:

Table 12. Regression analysis second hypothesis-Anova

\begin{tabular}{|c|c|c|c|c|c|c|c|c|c|}
\hline \multicolumn{10}{|c|}{ Model Summary $^{b}$} \\
\hline \multirow[b]{2}{*}{ Model } & \multirow[b]{2}{*}{$\mathrm{R}$} & \multirow[b]{2}{*}{$\begin{array}{c}\mathrm{R} \\
\text { Square }\end{array}$} & \multirow[b]{2}{*}{$\begin{array}{l}\text { Adjusted R } \\
\text { Square }\end{array}$} & \multirow[b]{2}{*}{$\begin{array}{l}\text { Std. Error of the } \\
\text { Estimate }\end{array}$} & \multicolumn{5}{|c|}{ Change Statistics } \\
\hline & & & & & $\begin{array}{l}\text { R Square } \\
\text { Change }\end{array}$ & $\begin{array}{c}\mathrm{F} \\
\text { Change }\end{array}$ & df1 & df2 & $\begin{array}{l}\text { Sig. F } \\
\text { Change }\end{array}$ \\
\hline 1 & $.508^{\mathrm{a}}$ & .258 & .232 & .773 & .258 & 9.977 & 4 & 115 & .000 \\
\hline
\end{tabular}

a. Predictors: (Constant), Willingness to Increase Sales on Its Investment Growth, Decrease Accounts payableon Enterprise, Willingness to Reduce Its Purchases on Investment Growth, Increase Accounts Receivable on Venture

b. Dependent Variable: Increase accounts receivable and decrease accounts payable

Table 13. Regression analysis second hypothesis-Anova

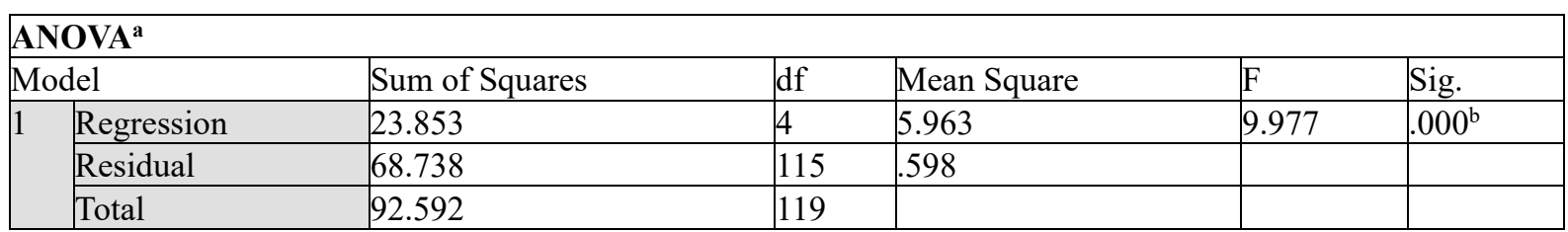

a. Dependent Variable: Increase accounts receivable and decrease accounts payable

b. Predictors: (Constant), Willingness to Increase Sales on Its Investment Growth, Decrease Accounts payableon Enterprise, Willingness to Reduce Its Purchases on Investment Growth, Increase Accounts Receivable on Venture

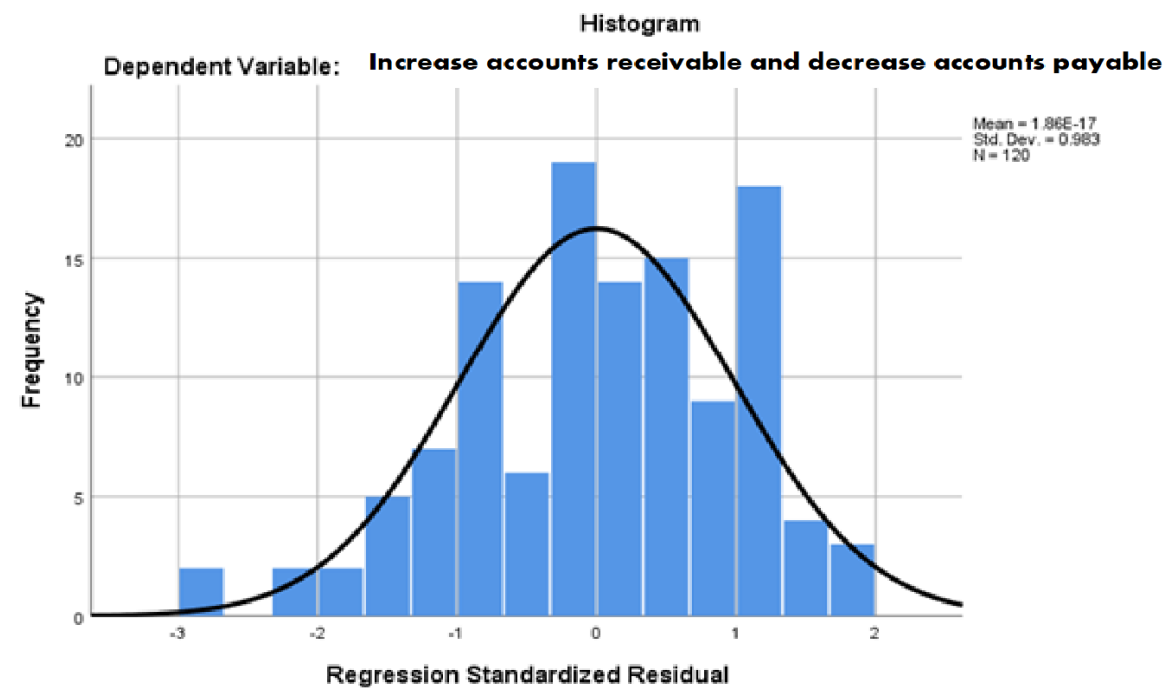

Chart 26. Regression analysis second hypothesis-Histogram 


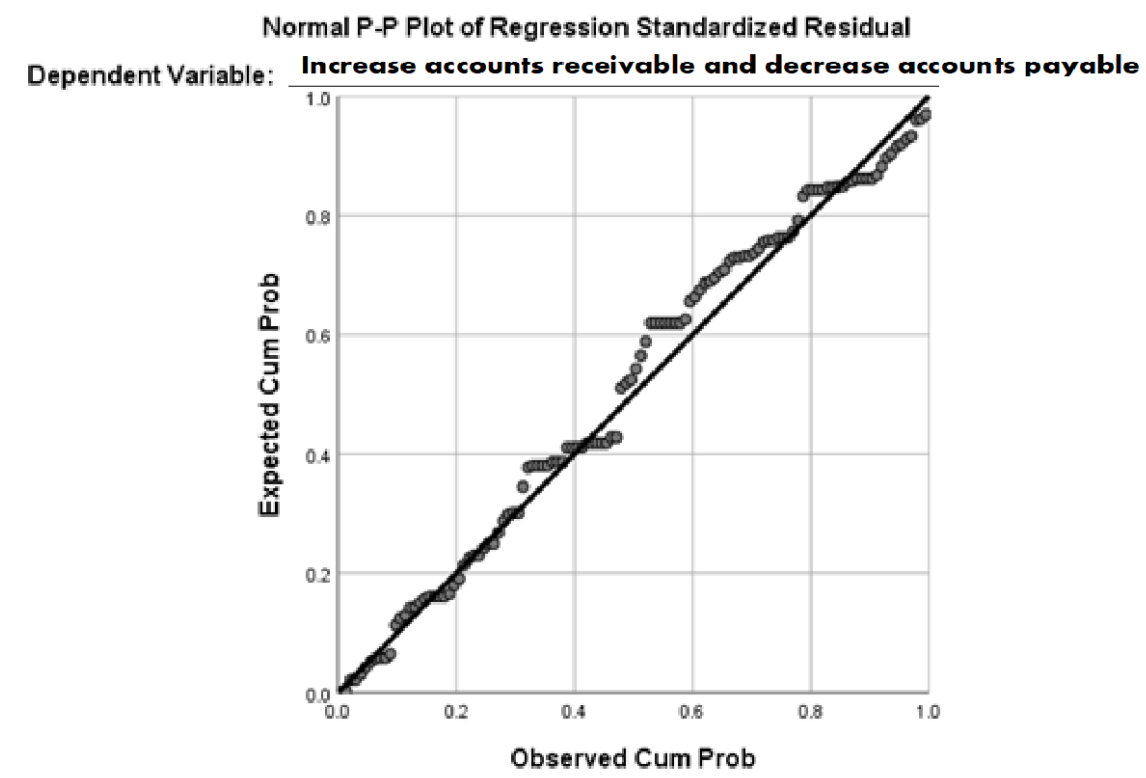

Chart 27. Regression analysis second hypothesis- Normal probability plot

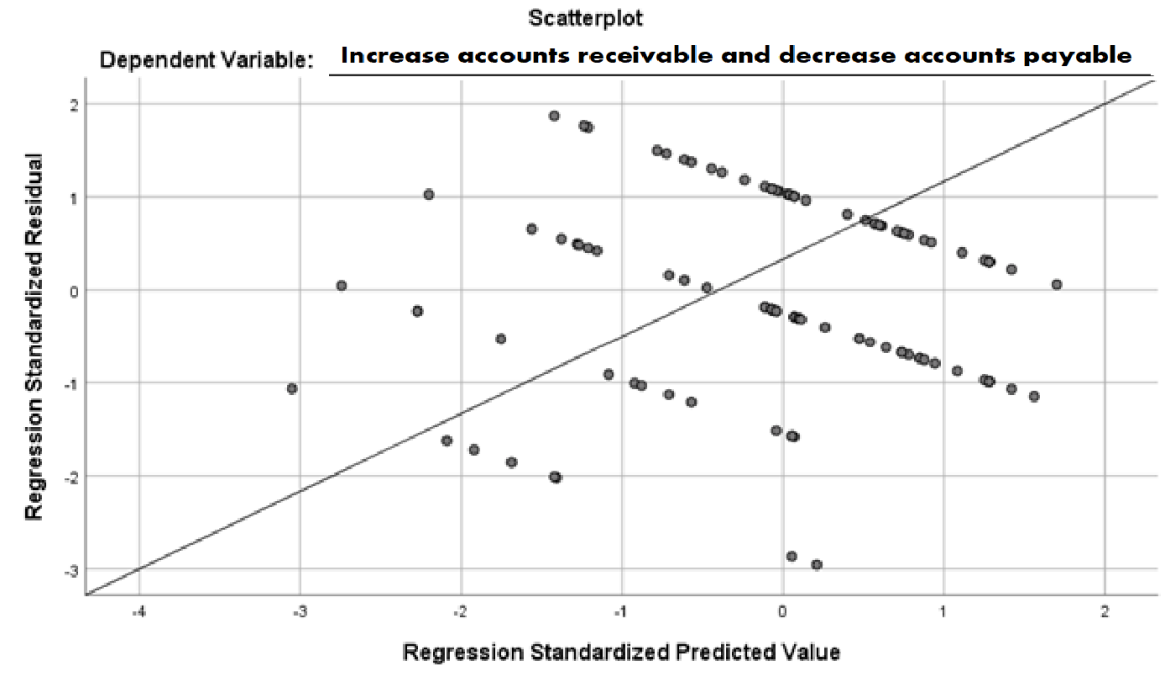

Chart 28. Regression analysis second hypothesisFor the second hypothesis the value of R Square turns out to be 0.258 and the significance level is 0 . This makes us understand that employees think that accounts receivable should be increased and accounts payablein the enterprise should be reduced, this can be determined or projected for $25.8 \%$

So there is a big impact of workers' desire for changes in financial investment plans. of investment, Addition of accounts receivable in the enterprise, mark a major impact. The value "Sig.000" confirms this result because $\mathrm{P}$ $<0.05$.

So, H2o Hypothesis: Increasing accounts receivable and decreasing accounts payablein the enterprise, would positively affect the enterprise, accepted according to the Program used SPSS and the model used: Regression. The null hypothesis is approved and the alternative hypothesis is rejected.

\section{Conclusions}

The financial analysis is performed with a detailed and objective purpose, for the evaluation and analysis of the results of past financial enterprises, and their forecasts for the future. Financial analysis is extremely important and should be applied when investing and in venture capital. 
With financial analysis, investors evaluate their investments in the company and try to detect possible mistakes of the existing management of the company. With financial analysis, investors try to identify undervalued companies, compared to the potential you have. Financial statement analysis is used by investors to forecast the company's future cash flows, or to reduce the unpredictability of future cash flows. With financial analysis, investors not only analyze and not only predict future cash flows, but they are predicting future liquidity situation, profitability, debt and company activities.

We conclude that Samsung from 2015 to 2018 has aimed to increase foreign investment. From the research we conclude that the workers emphasize that the increase of foreign investments, even if they bring high potential for the growth of bad debts, they still increase the tendency for the enterprise to gain growth potential. It has been observed from research that in order for this increase in foreign investment to work, the number of clients must increase. This has been seen with the increase in receivables and the decrease in accounts payable.

\section{Recommendations}

The recommendations generated are as follows:

1. Increase foreign investment as in research we note that the share of current and non-current assets in total assets is almost proportional and has increasing potential.

2. Maintain the same operating expenses as Samsung has done so far, and keep operating profit high as shown in the Income Statement.

3. Continue to increase receivables by increasing the potential for customer growth.

4. Keep the accounts payableas low as Samsung as participation within the liability structure has great momentum.

\section{References}

Wild, eds. (2004). Fundamentals of Fiannacial Management. Ohio: South-Western.

Brigham, Eugene F., \& Micheal, C. Ehrhardt, eds. (2005). Financial Management: Theory and Practice. Ohio: South-Western.

Weygandt, Keiso \& Kimmel, eds. (2008). Principles of Corporate Finance. Boston: McGraw-Hill/Irwin.

Atrill, \& McLaney (2002). Basic Marketing: A Global Managerial Approach. New York: McGraw-Hill.

Joy, Elly (2017). Thomas P., Cindy D. Edmonds, Bor-Yi Tsay, Philip R. Olds, and Nancy W.

Hermanson, Edwards and Salmonson (1989). Fundamental Managerial Accounting Concepts. Boston: McGrawHill/Irwin.

Hermanson, Roger H., James, Don Edwards, \& Salmonson, R. F. eds. (1989). Accounting Principles. Boston: BPI/IRWIN.

Audited Financial Statements- Samsung. (2010-2020). Retrieved from http://www.samsung.com/us/aboutsamsung/investor_relations/financial_information/financial_statement.ht $\mathrm{ml}$

\section{Copyrights}

Copyright for this article is retained by the author(s), with first publication rights granted to the journal.

This is an open-access article distributed under the terms and conditions of the Creative Commons Attribution license (http://creativecommons.org/licenses/by/4.0/). 